\title{
Evidence for nutrition transition in Kuwait: over-consumption of macronutrients and obesity
}

\author{
Sahar Zaghloul', Suad N Al-Hooti ${ }^{2, *}$, Nawal Al-Hamad ${ }^{3}$, Sameer Al-Zenki ${ }^{2}$, \\ Husam Alomirah ${ }^{2}$, Iman Alayan ${ }^{2}$, Hassan Al-Attar ${ }^{2}$, Amani Al-Othman ${ }^{2}$, \\ Entessar Al-Shami ${ }^{3}$, Mona Al-Somaie ${ }^{3}$ and Robert T Jackson ${ }^{4}$ \\ ${ }^{1}$ National Nutrition Institute, Cairo, Egypt: ${ }^{2}$ Biotechnology Department, Kuwait Institute for Scientific Research, \\ PO Box 24885, Safat, Kuwait 13109: ${ }^{3}$ Administration of Food and Nutrition, Ministry of Health, Kuwait: \\ ${ }^{4}$ Department of Nutrition and Food Science, University of Maryland, College Park, MD, USA
}

Submitted 21 November 2011: Final revision received 2 June 2012: Accepted 15 July 2012: First published online 14 September 2012

\begin{abstract}
Objectives: To describe nutrient intakes and prevalence of overweight and obesity in a nationally representative sample of Kuwaitis and to compare intakes with reference values.

Design: Cross-sectional, multistage stratified, cluster sample.

Settings: National nutrition survey covering all geographical areas of the country. Subjects: Kuwaitis ( $n$ 1704) between 3 and 86 years of age.

Results: Obesity was more prevalent among women than men (50\% and $70 \%$ for females aged $19-50$ years and $\geq 51$ years, respectively, v. $29 \%$ and $42 \%$ for their male counterparts). Boys were more obese than girls, with the highest obesity rate among those aged $9-13$ years $(37 \%$ and $24 \%$ of males and females, respectively). Energy intake was higher than the estimated energy requirements for almost half of Kuwaiti children and one-third of adults. The Estimated Average Requirement was exceeded by $78-100 \%$ of the recommendation for protein and carbohydrates. More than two-thirds of males aged $\geq 4$ years exceeded the Tolerable Upper Intake Level for Na. Conversely, less than $20 \%$ of Kuwaitis, regardless of age, consumed $100 \%$ or more of the Estimated Average Requirement for vitamin D, vitamin E, Ca, $n-3$ and $n-6$ fatty acids. Less than $20 \%$ of children met the recommended level for fibre.

Conclusions: Nutrition transition among Kuwaitis was demonstrated by the increased prevalence of obesity and overweight, increased intakes of energy and macronutrients and decreased intakes of fibre and micronutrients. Interventions to increase awareness about healthy foods combined with modifications in subsidy policies are clearly warranted to increase consumption of low-energy, nutrient-dense foods.
\end{abstract}

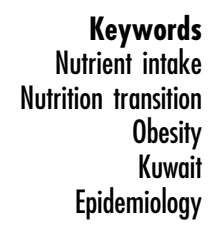

The relationship between food intake and noncommunicable diseases (NCD), where excessive and unbalanced intakes of energy, SFA, trans-fatty acids, salt and sugar are associated with nutrition-related NCD, is well established $^{(1)}$. The Gulf countries have the highest reported prevalence of NCD in the world ${ }^{(2)}$. Kuwait is a Gulf country with a total land area of $17818 \mathrm{~km}^{2}$ and a population of 3.3 million, of whom about $31 \%$ are Kuwaiti citizens. About $80 \%$ of adult Kuwaiti citizens ${ }^{(2,3)}$ are reported to be either overweight or obese and many of them suffer from one or more nutrition-related NCD such as diabetes $(15 \%)^{(3,4)}$, metabolic syndrome $(24 \%)^{(3)}$, CHD, hypertension $(26 \%)^{(5)}$ and/or dyslipidaemia $(33 \%)^{(5-7)}$. Kuwait has the highest childhood overweight problem among the Gulf countries and obesity is reportedly on the rise ${ }^{(2)}$. El-Bayoumy et $a l^{(8)}$ reported that $30 \cdot 7 \%$ and $14 \cdot 6 \%$ of children between the ages of 10 and 14 years were overweight and obese, respectively; while $43.3 \%$ and $21.3 \%$ of children between 14 and 19 years of age were overweight and obese, respectively ${ }^{(7)}$.

Before the discovery of oil, Kuwait's food supply was limited. Available foods consisted mainly of rice, dates, seafood, camel milk, sheep and goat meat and their by-products ${ }^{(9)}$. Meat was an indicator of wealth and the frequency of consumption of meat for the general population was weekly or monthly. Arabic coffee and milk represented main beverages while sweetened tea was served only on special occasions. Kuwait has experienced continued economic growth represented as increases in gross national income per capita (purchasing power parity) ${ }^{(10)}$, gross domestic product ${ }^{(11)}$ and population growth rate ${ }^{(12)}$. Economic growth in Kuwait has been 
accompanied by an increase in food availability, mainly of imported foods, which comprise $85 \%$ of all foods available in the market. Traditional foods have been replaced by foods typical of the Western diet through fast-food outlets and restaurants ${ }^{(9)}$. Increased food availability and reduced food prices (due to government subsidy) have contributed to increased energy intakes ${ }^{(13)}$. Increased daily consumption of energy-dense, nutrient-poor foods, increasing frequency of snacks and meals consumed away from home, shifts from drinking water and milk to drinking sugar-sweetened beverages and increased portion sizes are among the most commonly reported dietary changes associated with the nutrition transition, consequently increasing rates of nutritionrelated $\mathrm{NCD}^{(14-20)}$. Increased prevalences of obesity and nutrition-related NCD occur with increased intakes of energy, sugar, fat and protein and decreased intake of fibre ${ }^{(1,2,14-20)}$.

Except for a few studies reporting low fruit and vegetable intakes or increased consumption of fast foods and sugary snacks among Kuwaitis, mainly in children ${ }^{(21-24)}$, there is a paucity of national data on dietary intakes and assessment of compliance with dietary guidelines. Therefore the aims of the current study were to describe nutrient intakes and the prevalence of overweight and obesity in a nationally representative sample of Kuwaitis and to investigate the dietary determinants for nutrition transition by comparing dietary data with reference intake guidelines.

\section{Experimental methods}

\section{Sample}

This is a random representative national sample of Kuwaiti households. Kuwaiti households from the six governorates (Al Asema, Hawalli, Al Jahra, Al Farwania, Al Ahmadi and Mubarak Al Kabeer) were divided into eighty-two localities proportionate to Kuwaiti population density. Each locality was divided into clusters. Clusters of twenty households were selected using stratified sampling. Out of the total 5418 households contacted, 2862 households agreed to participate with a response rate of $53 \%$. At the household level, 1830 individuals ( $48 \%$ males and $52 \%$ females) were randomly selected from 545 separate Kuwaiti households from all six geographical strata, taking into consideration census gender distribution and age category. A screening form was completed per household and included demographic and socio-economic data and household composition. Data obtained were used to identify randomly selected individuals to survey from each age category and gender. Male household heads were recruited from odd-numbered households, while female household heads were selected from even-numbered households. In the case of having more than one participant within the same age group, both were selected if they were of different genders, or the male with odd serial number or the female with even serial number if both were of same gender.
Interviews were conducted at seven primary health-care clinics of the Ministry of Health located at various districts of Kuwait during the period July 2008 to November 2009 with a response rate of $24 \%$.

For the current paper, socio-economic, anthropometric and dietary data were analysed on a subsample of 1704 participants between the ages of 3 and 86 years for whom $24 \mathrm{~h}$ recalls were completed. Socio-economic, health and dietary data were collected on 655 children aged $1-18$ years and 1049 adults aged $\geq 19$ years. Anthropometric measurements and blood indices were taken. The study was approved by the Ethics Committee of the Kuwait Ministry of Health. Two consent forms were obtained, one from the head of the household and the other from each participant, including children, selected at the household level. Parents signed on behalf of their children. Consent forms were written in Arabic, as were the questionnaires. A raking method was used to calculate non-response-adjusted weights, producing a final set of person weights to perform data analyses.

\section{Antbropometric data}

Weight was measured using a Tanita 310 body composition analyser for children aged $\geq 7$ years and adults, while SECA scales were used for weight measurements of children $<7$ years of age. Measurements were taken to the closest $100 \mathrm{~g}$. A SECA 416 infantometer was used to obtain the length measurement of infants, while a SECA 214 stadiometer was used for measuring the height of older children and adults to the nearest $1 \mathrm{~cm}$.

BMI $\left(\mathrm{kg} / \mathrm{m}^{2}\right)$ was calculated by dividing weight in kilograms by the square of height in metres. Overweight and obesity were defined based on WHO standards. For adults, overweight was defined ${ }^{(25)}$ as $\mathrm{BMI} \geq 25 \cdot 0 \mathrm{~kg} / \mathrm{m}^{2}$ and obesity as BMI $\geq 30 \cdot 0 \mathrm{~kg} / \mathrm{m}^{2}$. For participants $\leq 5$ years of age, overweight was defined as BMI $Z$-score $\geq 2$ and obesity as BMI $Z$-score $\geq 3$ using WHO standards $^{(26)}$. For participants from 6 to 19 years of age ${ }^{(27)}$, overweight was defined as BMI $Z$-score $\geq 1$ and obesity as BMI $Z$-score $\geq 2$.

\section{Dietary data}

A single $24 \mathrm{~h}$ recall was collected from participants over 2 years of age using the multiple-pass method ${ }^{(28,29)}$ developed by the US Department of Agriculture and a food instruction booklet developed to standardize dietary data collection and reflect cultural and traditional eating behaviours ${ }^{(30)}$. In addition, food photographs and household measures (cups, spoons, etc.) were used to estimate portion sizes of foods. Food photographs were developed at the Kuwait Institute for Scientific Research where foods were cooked, served, weighed and photographed. Experienced dietitians were trained to collect the dietary data using the multiple-pass method and food instruction booklet. Mothers and/or other family members who were knowledgeable about the child's food intake were asked to 
provide types and quantities of food and beverages the child had consumed within the $24 \mathrm{~h}$ period preceding the interview. Adolescents responded for themselves. Given the lack of experience in collecting dietary $24 \mathrm{~h}$ recalls in Kuwait and the cultural context, we tested the quality of dietary data during the fieldwork for indicators of completeness and reasonability using a sample of 737 adults. For each adult participant, energy intake (EI) and BMR were calculated, the latter using the Schofield equations $^{(31)}$, and the ratio EI:BMR was determined. A cut-off value for EI:BMR of $<0.9$ was used to classify participants as under-reporters ${ }^{(32,33)}$. Furthermore, the estimated energy requirement (EER) was calculated according to the US Dietary Reference Intakes (DRI) ${ }^{(34)}$ from each participant's age, weight, height, gender and physical activity, and the ratio EI:EER computed. EI:EER provides an additional comparison capturing significant underreporting or over-reporting. These analyses revealed that $89 \%$ of males and $62 \%$ of females were adequate reporters. In addition, mean EI:BMR was 1.55 for adult females and 1.26 for adult males, denoting a mean underestimation percentage within acceptable cut-off points. We concluded that under-reporting is not likely to be a problem with the current study and in this cultural context.

\section{Dietary data processing}

The ESHA Food Processor software version $10 \cdot 3^{(35)}$ was used for dietary data entry after adding 103 chemically analysed local Kuwaiti composite dishes to the software database ${ }^{(36-41)}$. Recipes were created for an additional forty-six local foods reported in the $24 \mathrm{~h}$ recall. The nutrient composition of twenty breads and bakery products was obtained from Kuwait Flour Mills Company's nutrient analysis laboratory. Many commonly eaten food products in the market were investigated for nutrient content and adjusted in the nutrient database to reflect food fortification and enrichment in the Kuwaiti market. In the case of missing nutrient data for unanalysed food items, the closest similar food in the ESHA Food Processor program was selected to impute the missing nutrients.

The $24 \mathrm{~h}$ recalls were coded and foods closest in description and nutrient content were selected from US dietary databases. After initial data entry, a second coder checked each recall to verify accuracy. All recall surveys were rechecked by survey research supervisors. Dietary recalls were analysed for macronutrients, nine vitamins and seven minerals.

Nutrient intakes were compared with the US DRI since Kuwait does not have its own dietary guidelines. Kuwaitis' nutrition status was evaluated when appropriate against the Estimated Average Requirement (EAR), Adequate Intake (AI) and Acceptable Macronutrient Distribution Range (AMDR) developed by the Institute of Medicine's Food and Nutrition Board ${ }^{(34,42-48)}$ for all reported nutrients. The percentages of consumers meeting $100 \%$ or more of the appropriate DRI were calculated.

\section{Statistical analysis}

The SPSS statistical software package version 16 was used to calculate weighted means and standard errors of the nutrient intakes using individual weights developed based on census data and taking into account the complex sampling design. The percentage of energy from macronutrients and the percentage of participants who over-consumed energy and nutrients by age category and gender were estimated. Student's $t$ test was used to compare between male and female participants. The percentage of overweight and obese participants was estimated by age and gender. ANOVA was performed to test differences in mean intake by social factors at $95 \%$ confidence interval with a significance level of $P<0 \cdot 05$.

\section{Results}

\section{Description of the population}

As shown in Table 1 , more than half of all adult participants were female $(55 \cdot 3 \%)$ and most $(74 \cdot 1 \%)$ were married. Some $52.9 \%$ of adult participants had a monthly income of 1000-2000 Kuwaiti Dinars (approximately \$US 3500-7000), $7 \cdot 4 \%$ were either illiterate or functionally illiterate (can read and write but with no formal education),

Table 1 Characteristics of the sample of Kuwaiti adults ( $n$ 1049), 2009-2010

\begin{tabular}{|c|c|c|}
\hline Characteristic & $n$ & $\%$ \\
\hline \multicolumn{3}{|l|}{ Gender } \\
\hline Male & 469 & $44 \cdot 7$ \\
\hline Female & 580 & $55 \cdot 3$ \\
\hline \multicolumn{3}{|l|}{ Education } \\
\hline Illiteracy/functional illiteracy & 78 & $7 \cdot 4$ \\
\hline Less than high school & 239 & $22 \cdot 8$ \\
\hline High school/diploma & 433 & $41 \cdot 3$ \\
\hline More than high school & 299 & $28 \cdot 5$ \\
\hline \multicolumn{3}{|l|}{ Marital status } \\
\hline Married & 777 & $74 \cdot 1$ \\
\hline Single & 177 & $16 \cdot 9$ \\
\hline Divorced/widower & 95 & $9 \cdot 0$ \\
\hline \multicolumn{3}{|l|}{ Employment } \\
\hline Salary employed & 463 & $44 \cdot 1$ \\
\hline Work at home & 250 & $23 \cdot 8$ \\
\hline Retired & 212 & $20 \cdot 2$ \\
\hline Unemployed/student & 111 & $10 \cdot 7$ \\
\hline Self-employed & 12 & $1 \cdot 1$ \\
\hline \multicolumn{3}{|l|}{ Monthly family income (KD) } \\
\hline$<1000$ & 243 & $23 \cdot 2$ \\
\hline $1000-1499$ & 289 & $27 \cdot 6$ \\
\hline 1500-1999 & 265 & $25 \cdot 3$ \\
\hline$>2000$ & 252 & $24 \cdot 1$ \\
\hline \multicolumn{3}{|l|}{ BMI category } \\
\hline Underweight & 17 & $1 \cdot 6$ \\
\hline Overweight & 347 & $33 \cdot 1$ \\
\hline \multirow[t]{2}{*}{ Obese } & 452 & $43 \cdot 1$ \\
\hline & Mean & SD \\
\hline Weight (kg) & $79 \cdot 5$ & $19 \cdot 2$ \\
\hline Height $(\mathrm{cm})$ & $163 \cdot 7$ & $10 \cdot 8$ \\
\hline Waist circumference $(\mathrm{cm})$ & $96 \cdot 3$ & $16 \cdot 6$ \\
\hline
\end{tabular}

KD, Kuwaiti Dinars. 
$44 \cdot 1 \%$ were salary employed in either the government or private sector, and $23.8 \%$ of them worked at home (including housewives).

\section{Prevalence of overweight and obesity}

Weighted data revealed that mean BMI increased with age among males and females (Table 2). Overweight and obesity were more prevalent among male children ( $\leq 18$ years) compared with female children especially the 9-13 years age group. More than two-thirds of the adults were either overweight or obese, reaching a high of $78 \%$ and $93 \%$ in males and females aged $\geq 51$ years, respectively.

\section{Nutrient intakes}

Table 2 shows that a large percentage of Kuwaitis exhibited over-consumption of energy. However, that over-consumption decreased with age: $31 \cdot 5-72 \cdot 6 \%$ of children and $15 \cdot 5-31 \cdot 4 \%$ of adult participants exceeded the recommended energy requirements. The daily energy intake of children ranged from 5347 to $11068 \mathrm{~kJ}$ $(1278-2645 \mathrm{kcal})$, while adults consumed between 6078 and $10524 \mathrm{~kJ}(1454-2515 \mathrm{kcal})$. Males consumed more energy than females. Although macronutrient (protein, carbohydrates, fat) consumption fell within the AMDR for most ages, almost a third of the sample exceeded the upper limit of the AMDR for fat ( $35 \%$ of total energy) except for children aged $1-3$ years and adults aged $\geq 51$ years. On the other hand, $\geq 51$-year-old adults exceeded the upper limit of the AMDR for carbohydrates. Marked under-consumption of $n-3$ and $n-6$ fatty acids occurred across all age and gender groups, showing no gender differences.

Table 3 shows that the ranges of mean intake of carbohydrates, protein and fat for females were 184-264 g, 48-71 $\mathrm{g}$ and 44-75 g, respectively; corresponding values for males were $175-355 \mathrm{~g}, 48-110 \mathrm{~g}$ and $45-96 \mathrm{~g}$. The mean intake of cholesterol was less than the recommended $300 \mathrm{mg}$, except for males aged $19-50$ years. Mean fibre intake was less than the recommended AI value for all age groups among both males and females. Mean intake of fibre increased with age, with about a third of males and females aged $\geq 51$ years consuming $100 \%$ or more of the recommended amount of fibre for their age.

Excluding intakes of vitamins and minerals obtained from supplements, Figs 1 and 2 show the general inadequacy

Table 2 Prevalence of obesity, overweight and over-consumption of energy and macronutrients by age group and gender: nationally representative sample of Kuwaitis, 2009-2010

\begin{tabular}{|c|c|c|c|c|c|c|c|c|c|c|c|c|}
\hline \multirow{2}{*}{$\begin{array}{l}\text { Age (years) } \\
\text { Gender }\end{array}$} & \multicolumn{2}{|c|}{$1-3$} & \multicolumn{2}{|c|}{$4-8$} & \multicolumn{2}{|c|}{$9-13$} & \multicolumn{2}{|c|}{$14-18$} & \multicolumn{2}{|c|}{$19-50$} & \multicolumn{2}{|c|}{$\geq 51$} \\
\hline & $\begin{array}{l}\text { Male } \\
(n \text { 22) }\end{array}$ & $\begin{array}{c}\text { Female } \\
(n 19)\end{array}$ & $\begin{array}{c}\text { Male } \\
(n \text { 120) }\end{array}$ & $\begin{array}{l}\text { Female } \\
(n \text { 108) }\end{array}$ & $\begin{array}{c}\text { Male } \\
(n 111)\end{array}$ & $\begin{array}{c}\text { Female } \\
(n 94)\end{array}$ & $\begin{array}{l}\text { Male } \\
(n 95)\end{array}$ & $\begin{array}{c}\text { Female } \\
(n 86)\end{array}$ & $\begin{array}{c}\text { Male } \\
(n 315)\end{array}$ & $\begin{array}{l}\text { Female } \\
(n \text { 400) }\end{array}$ & $\begin{array}{c}\text { Male } \\
(n \text { 154) }\end{array}$ & $\begin{array}{l}\text { Female } \\
(n \text { 180) }\end{array}$ \\
\hline \multicolumn{13}{|l|}{ Anthropometry } \\
\hline Normal weight (\%) & $51 \cdot 1$ & $59 \cdot 9$ & $68 \cdot 1$ & $76 \cdot 9$ & 33.5 & $55 \cdot 5$ & $41 \cdot 8$ & $54 \cdot 7$ & $27 \cdot 9$ & $27 \cdot 3$ & $21 \cdot 6$ & $7 \cdot 0$ \\
\hline Overweight (\%) & $21 \cdot 1$ & $23 \cdot 2$ & $14 \cdot 1$ & $9 \cdot 4$ & $16 \cdot 7$ & $14 \cdot 6$ & $22 \cdot 7$ & $22 \cdot 4$ & $37 \cdot 9$ & $20 \cdot 3$ & $35 \cdot 4$ & $23 \cdot 2$ \\
\hline Obese (\%) & $18 \cdot 4$ & $11 \cdot 8$ & $6 \cdot 7$ & $10 \cdot 2$ & $36 \cdot 5$ & $24 \cdot 0$ & $25 \cdot 3$ & $17 \cdot 5$ & $29 \cdot 3$ & $49 \cdot 5$ & $42 \cdot 3$ & $69 \cdot 8$ \\
\hline \multicolumn{13}{|l|}{$\mathrm{BMI}\left(\mathrm{kg} / \mathrm{m}^{2}\right)$} \\
\hline Mean & $17 \cdot 0$ & $16 \cdot 6$ & $15 \cdot 4$ & $16 \cdot 1$ & $20 \cdot 6$ & $19 \cdot 7$ & $23 \cdot 5$ & $23 \cdot 6$ & $28 \cdot 1$ & $30 \cdot 0$ & $29 \cdot 3$ & 33.2 \\
\hline SE & 0.3 & 0.2 & 0.3 & 0.3 & 0.9 & 0.9 & 0.9 & 0.8 & 0.7 & 0.6 & 0.7 & 0.6 \\
\hline \multicolumn{13}{|l|}{ Diet } \\
\hline \multicolumn{13}{|l|}{ Energy $(\mathrm{kJ} / \mathrm{d})$} \\
\hline Mean & 5347 & 5595 & 7414 & 6796 & 9356 & 8336 & 11068 & 7599 & 10524 & 7144 & 8343 & 6078 \\
\hline SE & 385 & 625 & 308 & 226 & 444 & 387 & 431 & 352 & 267 & 174 & 354 & 296 \\
\hline \multicolumn{13}{|l|}{ Energy $(\mathrm{kcal} / \mathrm{d})$} \\
\hline Mean & 1278 & 1337 & 1772 & 1624 & 2236 & 1992 & 2645 & 1816 & 2515 & 1707 & 1994 & 1453 \\
\hline SE & 92 & 149 & 74 & 54 & 106 & 92 & 103 & 84 & 64 & 42 & 85 & 71 \\
\hline $\begin{array}{l}\% \text { over-consumption relative to EER } \\
\text { Protein }\end{array}$ & $40 \cdot 9$ & $57 \cdot 6$ & $61 \cdot 9$ & $72 \cdot 6$ & $43 \cdot 5$ & $63 \cdot 3$ & $31 \cdot 5$ & $50 \cdot 8$ & $31 \cdot 4$ & $28 \cdot 5$ & $25 \cdot 2$ & $15 \cdot 5$ \\
\hline$\%$ energy $/ \mathrm{d}$ & 14 & 13 & 15 & 15 & 15 & 14 & 15 & 15 & 17 & 16 & 17 & 18 \\
\hline$\%$ met AMDR & $82 \cdot 4$ & $90 \cdot 0$ & $93 \cdot 5$ & $90 \cdot 7$ & $86 \cdot 3$ & $86 \cdot 0$ & $92 \cdot 4$ & $89 \cdot 3$ & $99 \cdot 6$ & $98 \cdot 4$ & $100 \cdot 0$ & $96 \cdot 4$ \\
\hline$\%$ above AMDR & $17 \cdot 6$ & $10 \cdot 0$ & 0 & 0 & 0 & 0 & 0 & 0 & 0.4 & 0.7 & 0 & 3.6 \\
\hline \multicolumn{13}{|l|}{ Carbohydrates } \\
\hline$\%$ energy/d & 56 & 57 & 52 & 53 & 53 & 53 & 54 & 53 & 53 & 54 & 57 & 56 \\
\hline$\%$ met AMDR & $72 \cdot 1$ & $50 \cdot 7$ & $64 \cdot 6$ & $74 \cdot 2$ & $69 \cdot 8$ & $69 \cdot 5$ & $62 \cdot 8$ & $67 \cdot 9$ & $57 \cdot 9$ & $64 \cdot 1$ & $49 \cdot 0$ & $56 \cdot 7$ \\
\hline$\%$ above AMDR & $10 \cdot 0$ & $31 \cdot 8$ & $12 \cdot 7$ & $8 \cdot \overline{3}$ & $13 \cdot 8$ & $13 \cdot 6$ & $17 \cdot 2$ & $13 \cdot 3$ & $15 \cdot 6$ & $16 \cdot 4$ & $30 \cdot 4$ & $27 \cdot 6$ \\
\hline \multicolumn{13}{|l|}{ Fat } \\
\hline$\%$ energy $/ \mathrm{d}$ & 30 & 30 & 33 & 32 & 32 & 33 & 31 & 32 & 30 & 30 & 26 & 26 \\
\hline$\%$ met AMDR & $46 \cdot 4$ & $30 \cdot 7$ & $41 \cdot 7$ & $53 \cdot 7$ & $42 \cdot 3$ & $45 \cdot 7$ & $41 \cdot 8$ & $47 \cdot 3$ & $53 \cdot 7$ & $59 \cdot 2$ & $47 \cdot 4$ & $58 \cdot 3$ \\
\hline$\%$ above AMDR & $13 \cdot 6$ & $15 \cdot 3$ & $40 \cdot 8$ & $36 \cdot 3$ & $39 \cdot 7$ & $38 \cdot 7$ & $31 \cdot 4$ & $37 \cdot 8$ & $33 \cdot 6$ & $32 \cdot 9$ & $22 \cdot 9$ & $16 \cdot 7$ \\
\hline \multicolumn{13}{|l|}{$n-3$ Fatty acids } \\
\hline$\%$ energy $/$ d & $1 \cdot 1$ & 0.6 & $0 \cdot 7$ & 0.8 & $0 \cdot 8$ & 0.9 & 0.9 & $1 \cdot 3$ & $1 \cdot 1$ & $1 \cdot 1$ & $1 \cdot 0$ & $1 \cdot 1$ \\
\hline$\%$ met AMDR & $8 \cdot 7$ & 0 & $3 \cdot 1$ & $3 \cdot 3$ & $2 \cdot 2$ & $5 \cdot 1$ & $3 \cdot 6$ & $6 \cdot 7$ & $6 \cdot 1$ & $6 \cdot 0$ & $3 \cdot 1$ & $3 \cdot 6$ \\
\hline$\%$ above AMDR & 0 & 0 & 0 & 0 & $0 . \overline{8}$ & 0 & $1 \cdot 1$ & 0 & 0.8 & 0.8 & 0 & $1 \cdot 3$ \\
\hline \multicolumn{13}{|l|}{$n-6$ Fatty acids } \\
\hline$\%$ energy/d & $2 \cdot 0$ & $2 \cdot 4$ & $4 \cdot 0$ & 3.5 & $3 \cdot 7$ & 3.6 & 3.6 & $2 \cdot 8$ & $3 \cdot 2$ & $3 \cdot 8$ & $3 \cdot 3$ & $3 \cdot 3$ \\
\hline$\%$ met AMDR & $12 \cdot 0$ & $4 \cdot 2$ & $16 \cdot 5$ & $22 \cdot 5$ & $28 \cdot 1$ & $25 \cdot 9$ & $28 \cdot 7$ & $16 \cdot 7$ & $19 \cdot 5$ & $20 \cdot 7$ & $16 \cdot 1$ & $9 \cdot 2$ \\
\hline$\%$ above AMDR & 0 & $3 \cdot 1$ & $7 \cdot 1$ & $2 \cdot 7$ & $1 \cdot 3$ & 0.5 & $2 \cdot 3$ & 0.9 & $1 \cdot 1$ & $4 \cdot 7$ & $4 \cdot 4$ & $4 \cdot \overline{5}$ \\
\hline
\end{tabular}

EER, estimated energy requirement; AMDR, Acceptable Macronutrient Distribution Range. 
Table 3 Weighted means, standard errors and percentage consumption of macronutrients in excess of the Dietary Reference Intakest by age group and gender: nationally representative sample of Kuwaitis, 2009-2010

\begin{tabular}{|c|c|c|c|c|c|c|c|c|c|c|c|c|}
\hline \multirow{2}{*}{$\begin{array}{l}\text { Age (years) } \\
\text { Gender }\end{array}$} & \multicolumn{2}{|c|}{$1-3$} & \multicolumn{2}{|c|}{$4-8$} & \multicolumn{2}{|c|}{$9-13$} & \multicolumn{2}{|c|}{$14-18$} & \multicolumn{2}{|c|}{$19-50$} & \multicolumn{2}{|c|}{$\geq 51$} \\
\hline & Male & Female & Male & Female & Male & Female & Male & Female & Male & Female & Male & Female \\
\hline \multicolumn{13}{|l|}{ Protein $(\mathrm{g})$} \\
\hline EAR & 11 & 11 & 15 & 15 & 27 & 28 & 44 & 38 & 46 & 38 & 46 & 38 \\
\hline Mean & $47 \cdot 9$ & $47 \cdot 8$ & $69 \cdot 8$ & $62 \cdot 5$ & $87 \cdot 1$ & $70 \cdot 8$ & $99 \cdot 1$ & 68.9 & $110 \cdot 1$ & $67 \cdot 4$ & $90 \cdot 0$ & $67 \cdot 2$ \\
\hline SE & $5 \cdot 6$ & $7 \cdot 1$ & $3 \cdot 3$ & $2 \cdot 8$ & $6 \cdot 3$ & $4 \cdot 2$ & $4 \cdot 9$ & $3 \cdot 3$ & $4 \cdot 1$ & $2 \cdot 3$ & $4 \cdot 2$ & $4 \cdot 2$ \\
\hline$\%$ exceed & $100 \cdot 0$ & $100 \cdot 0$ & $99 \cdot 4$ & $100 \cdot 0$ & $94 \cdot 8$ & $92 \cdot 5$ & $89 \cdot 9$ & $77 \cdot 7$ & $91 \cdot 0$ & $80 \cdot 7$ & $82 \cdot 6$ & $83 \cdot 6$ \\
\hline \multicolumn{13}{|c|}{ Carbohydrates (g) } \\
\hline EAR & 100 & 100 & 100 & 100 & 100 & 100 & 100 & 100 & 100 & 100 & 100 & 100 \\
\hline Mean & $174 \cdot 8$ & $184 \cdot 1$ & $226 \cdot 1$ & $215 \cdot 1$ & $295 \cdot 5$ & $264 \cdot 2$ & $355 \cdot 4$ & $240 \cdot 2$ & $327 \cdot 6$ & $228 \cdot 4$ & $276 \cdot 0$ & $201 \cdot 4$ \\
\hline SE & $12 \cdot 2$ & $13 \cdot 9$ & $10 \cdot 2$ & $9 \cdot 4$ & 14 & $11 \cdot 2$ & $17 \cdot 1$ & $12 \cdot 3$ & $8 \cdot 8$ & $8 \cdot 4$ & $12 \cdot 1$ & $9 \cdot 5$ \\
\hline$\%$ exceed & $95 \cdot 1$ & $95 \cdot 3$ & $95 \cdot 1$ & $96 \cdot 9$ & $97 \cdot 2$ & $97 \cdot 8$ & $99 \cdot 2$ & $96 \cdot 3$ & $98 \cdot 4$ & $90 \cdot 9$ & $97 \cdot 1$ & $92 \cdot 6$ \\
\hline \multicolumn{13}{|l|}{ Fat (g) } \\
\hline EAR & ND & ND & ND & ND & ND & ND & ND & ND & ND & ND & ND & ND \\
\hline Mean & $45 \cdot 1$ & $47 \cdot 5$ & $67 \cdot 2$ & $59 \cdot 2$ & $81 \cdot 1$ & $75 \cdot 4$ & $95 \cdot 7$ & $67 \cdot 2$ & $87 \cdot 3$ & $60 \cdot 7$ & $61 \cdot 8$ & $44 \cdot 4$ \\
\hline SE & $4 \cdot 1$ & $8 \cdot 2$ & $3 \cdot 7$ & $2 \cdot 2$ & $4 \cdot 4$ & $4 \cdot 6$ & $5 \cdot 6$ & $3 \cdot 6$ & $3 \cdot 1$ & $2 \cdot 1$ & $3 \cdot 9$ & $3 \cdot 4$ \\
\hline \multicolumn{13}{|l|}{ Fibre (g) } \\
\hline EAR & 19 & 19 & 25 & 25 & 31 & 26 & 38 & 26 & 38 & 25 & 30 & 21 \\
\hline Mean & $8 \cdot 5$ & $13 \cdot 8$ & $14 \cdot 3$ & $11 \cdot 7$ & $18 \cdot 1$ & 16 & $20 \cdot 5$ & $16 \cdot 9$ & $26 \cdot 2$ & $20 \cdot 5$ & $29 \cdot 7$ & $20 \cdot 3$ \\
\hline SE & $1 \cdot 1$ & $1 \cdot 6$ & $1 \cdot 1$ & 0.7 & $1 \cdot 6$ & $1 \cdot 3$ & 1.9 & $1 \cdot 6$ & 1.5 & 0.7 & $2 \cdot 0$ & $1 \cdot 3$ \\
\hline$\%$ exceed & $1 \cdot 3$ & $7 \cdot 9$ & $13 \cdot 6$ & $5 \cdot 7$ & $14 \cdot 7$ & $13 \cdot 4$ & $9 \cdot 0$ & $19 \cdot 3$ & $19 \cdot 7$ & $27 \cdot 5$ & $38 \cdot 6$ & $33 \cdot 4$ \\
\hline \multicolumn{13}{|c|}{ Cholesterol (mg) } \\
\hline EAR & 300 & 300 & 300 & 300 & 300 & 300 & 300 & 300 & 300 & 300 & 300 & 300 \\
\hline Mean & $141 \cdot 9$ & $126 \cdot 4$ & $270 \cdot 0$ & $211 \cdot 3$ & $272 \cdot 7$ & $220 \cdot 2$ & 277 & $217 \cdot 1$ & 345.5 & $220 \cdot 5$ & $256 \cdot 7$ & $187 \cdot 8$ \\
\hline SE & $32 \cdot 8$ & $24 \cdot 2$ & $23 \cdot 5$ & $20 \cdot 5$ & $20 \cdot 9$ & $29 \cdot 8$ & $24 \cdot 5$ & $28 \cdot 6$ & $20 \cdot 7$ & $16 \cdot 0$ & $26 \cdot 3$ & $21 \cdot 0$ \\
\hline$\%$ exceed & $4 \cdot 0$ & $2 \cdot 2$ & $33 \cdot 8$ & $21 \cdot 3$ & $35 \cdot 0$ & $25 \cdot 3$ & $29 \cdot 6$ & $18 \cdot 3$ & $47 \cdot 9$ & $21 \cdot 9$ & 30.5 & $20 \cdot 1$ \\
\hline
\end{tabular}

EAR, Estimated Average Requirement; ND, not defined.

tPercentage of participants who consumed $\geq 100 \%$ of the EAR.

$\ddagger$ Percentage of participants who consumed $\geq 100 \%$ of the Adequate Intake for fibre $(\mathrm{g})$.

$\S$ Percentage of participants who consumed $\geq 300 \mathrm{mg} / \mathrm{d}$.

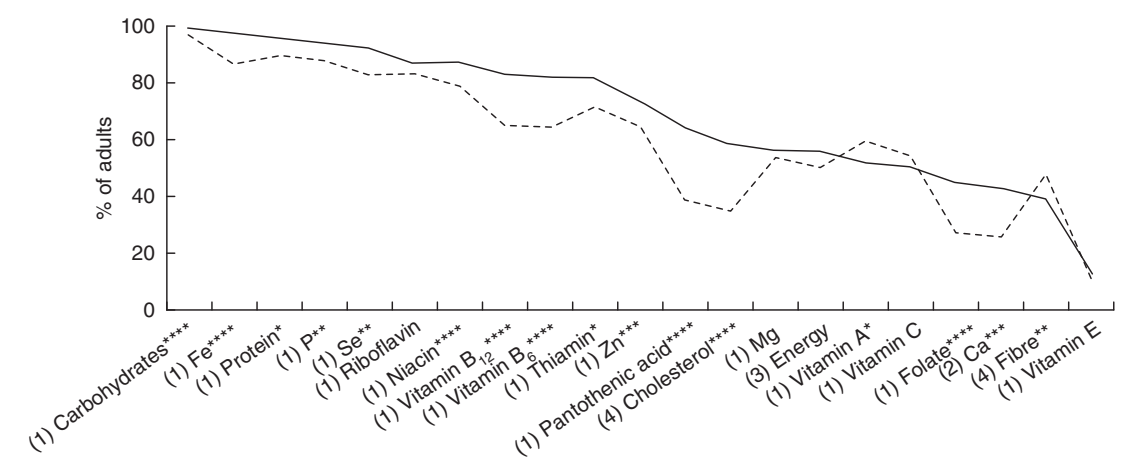

Fig. 1 Percentage of Kuwaiti adults meeting the Dietary Reference Intakes ( $1=$ Estimated Average Requirement, $2=$ Adequate Intake; 3 = estimated energy requirement, $4=$ Acceptable Macronutrient Distribution Range) by gender ( - , males; - - -, females), 2009-2010. Significance levels for comparisons by gender: ${ }^{*} P<0 \cdot 05,{ }^{\star \star} P<0 \cdot 01,{ }^{\star \star \star} P<0.001,{ }^{\star \star \star \star} P<0.0001$

of nutrient intakes among adults and children. Recommended intakes of vitamin E, fibre, folate and Ca were met by less than half of the adult population with a significantly low intake of most nutrients by women. Fewer than $10 \%$ of adults met the EAR for vitamin E, and less than $50 \%$ for fibre, $\mathrm{Ca}$, folate and vitamin C. Similarly, low intakes were observed among children; however, significant gender differences were observed only for vitamin $\mathrm{B}_{12}, \mathrm{Zn}$, Ca and P. More adult Kuwaitis met the recommended EAR values for $\mathrm{Zn}, \mathrm{P}$ and $\mathrm{Mg}$ than did children.

Table 4 shows mean intake of vitamins by age category and gender. Results revealed extremely low percentages of participants consuming $100 \%$ or more of the EAR for vitamin D, vitamin E and folate $(<2 \%$ for vitamin $\mathrm{D}$ and $<10 \%$ for vitamin E). Prevalence of low serum levels of 25-hydroxyvitamin D $(<25 \mathrm{nmol} / \mathrm{l})$ was also found among the participants $(17 \%$ for males and 33\% for females), with the prevalence being significantly higher among females than males (data are not shown).

Conversely, mean intakes of vitamin A, vitamin $\mathrm{C}$, thiamin, riboflavin, niacin, vitamin $\mathrm{B}_{6}$ and vitamin $\mathrm{B}_{12}$ were greater than the EAR values across all age categories in both males and females. Vitamins $\mathrm{A}, \mathrm{C}$ and $\mathrm{B}_{12}$ were 


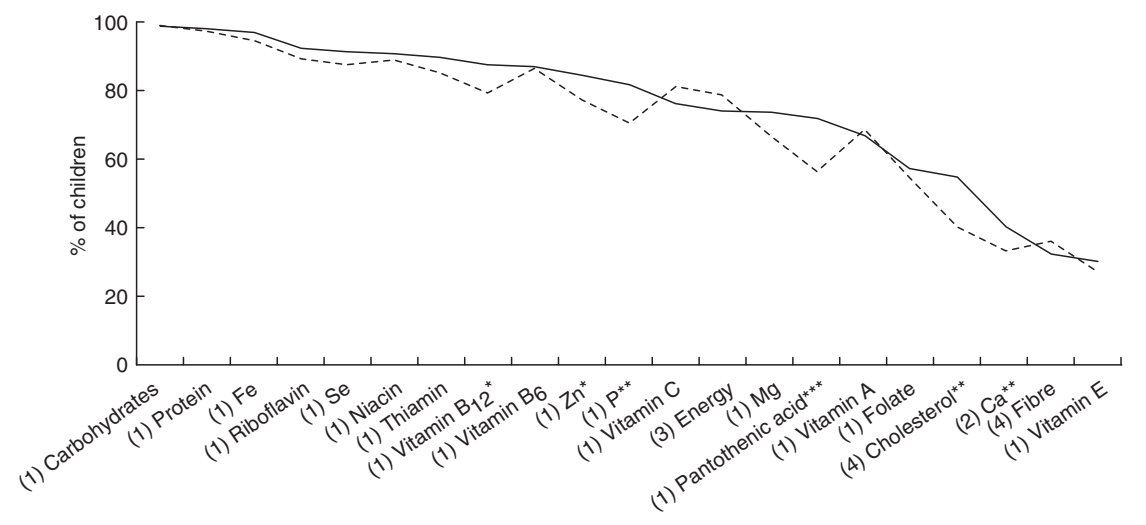

Fig. 2 Percentage of Kuwaiti children meeting the Dietary Reference Intakes ( 1 = Estimated Average Requirement, $2=$ Adequate Intake, 3 = estimated energy requirement, 4 = Acceptable Macronutrient Distribution Range) by gender ( - , males; - - -, females), 2009-2010. Significance levels for comparisons by gender: ${ }^{\star} P<0 \cdot 05,{ }^{\star \star} P<0 \cdot 01,{ }^{\star \star \star} P<0.001$

measured in serum and the majority of participants had all vitamin and trace element levels within the normal range (data not shown).

Table 5 shows the mean intake of minerals by age category and gender. Mean Fe intake for males was two to three times the recommended value. On the other hand, the mean $\mathrm{Ca}$ intake was markedly less than the recommended amount for both males and females across different age groups. The lowest mean $\mathrm{Ca}$ intakes were $543.8 \mathrm{mg}$ and $518.7 \mathrm{mg}$ for females aged 9-13 years and 14-18 years, respectively.

Females 14-18 years of age reported the lowest percentage of those consuming $100 \%$ or more of the EAR for $\mathrm{Ca}, \mathrm{Mg}$ and $\mathrm{P}$, although $50 \%$ of them consumed $100 \%$ or more of their energy requirement. Mean intake of $\mathrm{Zn}$ was low only among females aged $14-18$ years $(6 \cdot 8 \mathrm{mg})$. Mean intake of $\mathrm{Na}$ exceeded the Tolerable Upper Intake Level for all age groups. Salt added at the table was not quantified in the survey.

Tables 6-8 show mean macronutrient, vitamin and mineral intakes by education, occupation, marital status and family income among adults. Males consumed significantly more nutrients than females. Mean nutrient intakes were significantly highest among the self-employed compared with other occupations and lowest among illiterate and functionally illiterate participants compared with those of higher educational attainment. Surprisingly, mean nutrient intakes did not differ by family income except for riboflavin and niacin. Mean nutrient intakes differed significantly by marital status but the direction of differences was inconsistent.

\section{Discussion}

The present study is the first to our knowledge to describe nutrient intakes of a nationally representative sample of Kuwaitis based on actual food consumption and not on estimated food availability. The large percentage of the population which over-consumes energy and energyyielding nutrients, coupled with the high prevalence of obesity and overweight, clearly show that the nutrition transition is extant in Kuwait ${ }^{(49)}$. Almost half of the population consumed more than their energy, protein and carbohydrate requirements. The range of mean energy intakes was $5347-11068 \mathrm{~kJ}(1278-2645 \mathrm{kcal})$ for males and $5595-8336 \mathrm{~kJ}(1337-1992 \mathrm{kcal})$ for females. As expected, these empirically derived findings are lower than those estimated in the FAOSTAT data set for total energy $(13010 \mathrm{~kJ}$ or $3108 \mathrm{kcal})$, carbohydrates $(100 \mathrm{~g})$, protein $(92 \mathrm{~g})$ and fat $(114 \mathrm{~g})^{(14,50)}$. This difference is due the fact that FAOSTAT is an estimation of energy intake based on aggregated data sources. We expect our data set to contain age and gender differences contributing to the observed discrepancies in addition to other uncalculated losses.

The prevalence of overweight and obesity was high across almost all age groups, with the highest prevalence being seen among women $\geq 20$ years of age. This finding is consistent with previous reports ${ }^{(2,7,51)}$. Our results showed a slightly higher prevalence of obesity among adults in the same age range compared with those reported by Al Rashdan and Nesef ${ }^{(3)}$, who found 36.4\% of adult males to be obese and $47.9 \%$ of adult females. For children and adolescents, obesity prevalence ranged from $7 \%$ to $37 \%$ for males and from $10 \%$ to $24 \%$ for females. The comparison of childhood obesity rates with those found in previous studies is difficult because of the use of different cut-offs and standards. However, it is worth noting that $\mathrm{Ng}$ et $a l .{ }^{(2)}$ reported increased obesity prevalence for all children in Kuwait compared with the other Gulf countries and estimated an increased rate of $1.7 \%$ and $3.9 \%$ among women and men between 30 and 60 years of age, respectively.

Our study also showed over-consumption of $\mathrm{Na}$ and cholesterol at an early age, which may help to explain the high prevalence and early onset of CVD among Kuwaitis $^{(5,6)}$. Additionally, the high $\mathrm{Na}$ intake is consistent 
Table 4 Weighted means, standard errors and percentage of consumption of vitamins in excess of the Dietary Reference Intakes by age group and gender: nationally representative sample of Kuwaitis, 2009-2010

\begin{tabular}{|c|c|c|c|c|c|c|c|c|c|c|c|c|}
\hline \multirow{2}{*}{$\begin{array}{l}\text { Age (years) } \\
\text { Gender }\end{array}$} & \multicolumn{2}{|c|}{$1-3$} & \multicolumn{2}{|c|}{$4-8$} & \multicolumn{2}{|c|}{$9-13$} & \multicolumn{2}{|c|}{$14-18$} & \multicolumn{2}{|c|}{$19-50$} & \multicolumn{2}{|c|}{$\geq 51$} \\
\hline & Male & Female & Male & Female & Male & Female & Male & Female & Male & Female & Male & Female \\
\hline \multicolumn{13}{|c|}{ Vitamin A ( $\mu \mathrm{g} R E)$} \\
\hline EAR & 210 & 210 & 275 & 275 & 445 & 420 & 630 & 485 & 625 & 500 & 625 & 500 \\
\hline Mean & $328 \cdot 9$ & $292 \cdot 3$ & $524 \cdot 5$ & 555.9 & $744 \cdot 4$ & $777 \cdot 1$ & $588 \cdot 7$ & $924 \cdot 5$ & $867 \cdot 2$ & $1021 \cdot 1$ & $1183 \cdot 9$ & $995 \cdot 8$ \\
\hline SE & $35 \cdot 7$ & $25 \cdot 4$ & $57 \cdot 9$ & $101 \cdot 4$ & $138 \cdot 3$ & $218 \cdot 9$ & $67 \cdot 4$ & $178 \cdot 1$ & $105 \cdot 0$ & $137 \cdot 4$ & $141 \cdot 0$ & $114 \cdot 8$ \\
\hline$\%$ exceed & $23 \cdot 3$ & $19 \cdot 1$ & $60 \cdot 6$ & $60 \cdot 9$ & $51 \cdot 1$ & $39 \cdot 1$ & $31 \cdot 0$ & $50 \cdot 6$ & $39 \cdot 5$ & $45 \cdot 8$ & $48 \cdot 3$ & 45.8 \\
\hline \multicolumn{13}{|c|}{ Vitamin E (mg) } \\
\hline EAR & 5 & 5 & 6 & 6 & 9 & 9 & 12 & 12 & 12 & 12 & 12 & 12 \\
\hline Mean & $2 \cdot 5$ & $2 \cdot 8$ & $4 \cdot 7$ & $3 \cdot 5$ & $4 \cdot 8$ & $4 \cdot 2$ & $5 \cdot 4$ & $4 \cdot 1$ & $5 \cdot 3$ & 1.5 & 4.9 & $4 \cdot 8$ \\
\hline SE & $0 \cdot 3$ & 0.5 & 0.8 & $0 \cdot 3$ & $0 \cdot 4$ & $0 \cdot 3$ & 0.5 & $0 \cdot 4$ & $0 \cdot 3$ & $0 \cdot 2$ & $0 \cdot 3$ & $0 \cdot 2$ \\
\hline$\%$ exceed & $1 \cdot 6$ & $5 \cdot 1$ & $15 \cdot 4$ & $12 \cdot 0$ & $9 \cdot 6$ & $5 \cdot 7$ & $7 \cdot 1$ & $1 \cdot 9$ & $5 \cdot 8$ & $4 \cdot 6$ & $4 \cdot 4$ & $4 \cdot 1$ \\
\hline \multicolumn{13}{|l|}{ Thiamin (mg) } \\
\hline EAR & 0.4 & $0 \cdot 4$ & 0.5 & 0.5 & $0 \cdot 7$ & $0 \cdot 7$ & $1 \cdot 0$ & $0 \cdot 9$ & $1 \cdot 0$ & $0 \cdot 9$ & $1 \cdot 0$ & $0 \cdot 9$ \\
\hline Mean & $1 \cdot 0$ & $1 \cdot 4$ & $2 \cdot 2$ & $1 \cdot 8$ & $2 \cdot 0$ & $1 \cdot 4$ & $2 \cdot 6$ & 1.5 & $2 \cdot 8$ & 1.5 & $3 \cdot 4$ & $1 \cdot 6$ \\
\hline SE & $0 \cdot 3$ & 0.6 & 0.4 & $0 \cdot 3$ & $0 \cdot 3$ & $0 \cdot 2$ & 0.5 & $0 \cdot 3$ & $0 \cdot 3$ & $0 \cdot 2$ & 0.6 & $0 \cdot 2$ \\
\hline$\%$ exceed & $21 \cdot 8$ & $25 \cdot 0$ & $83 \cdot 9$ & $82 \cdot 8$ & $76 \cdot 5$ & $73 \cdot 9$ & $68 \cdot 7$ & $40 \cdot 0$ & $66 \cdot 7$ & $54 \cdot 2$ & $70 \cdot 5$ & $45 \cdot 3$ \\
\hline \multicolumn{13}{|c|}{ Riboflavin (mg) } \\
\hline EAR & 0.4 & $0 \cdot 4$ & 0.5 & 0.5 & $0 \cdot 8$ & $0 \cdot 8$ & $1 \cdot 1$ & $0 \cdot 9$ & $1 \cdot 1$ & $0 \cdot 9$ & $1 \cdot 1$ & $0 \cdot 9$ \\
\hline Mean & $1 \cdot 3$ & $1 \cdot 2$ & $1 \cdot 5$ & 1.5 & $1 \cdot 8$ & $1 \cdot 3$ & $1 \cdot 8$ & $1 \cdot 3$ & $1 \cdot 8$ & $1 \cdot 3$ & $1 \cdot 8$ & 1.3 \\
\hline SE & 0.2 & $0 \cdot 1$ & $0 \cdot 1$ & $0 \cdot 1$ & $0 \cdot 1$ & $0 \cdot 1$ & $0 \cdot 1$ & $0 \cdot 9$ & $0 \cdot 1$ & $0 \cdot 1$ & 0.2 & $0 \cdot 1$ \\
\hline$\%$ exceed & $27 \cdot 7$ & $26 \cdot 3$ & $91 \cdot 0$ & $91 \cdot 2$ & $86 \cdot 2$ & $75 \cdot 6$ & $77 \cdot 7$ & $56 \cdot 3$ & $77 \cdot 6$ & $68 \cdot 2$ & $73 \cdot 7$ & $69 \cdot 3$ \\
\hline \multicolumn{13}{|l|}{ Niacin (mg) } \\
\hline EAR & 5 & 5 & 6 & 6 & 9 & 9 & 12 & 11 & 12 & 11 & 12 & 11 \\
\hline Mean & $12 \cdot 6$ & $13 \cdot 1$ & $18 \cdot 1$ & $18 \cdot 3$ & $22 \cdot 8$ & $18 \cdot 9$ & $26 \cdot 3$ & $17 \cdot 7$ & $26 \cdot 6$ & $17 \cdot 4$ & $22 \cdot 4$ & $15 \cdot 2$ \\
\hline SE & $2 \cdot 4$ & $1 \cdot 4$ & $1 \cdot 2$ & $1 \cdot 1$ & $2 \cdot 2$ & $1 \cdot 3$ & $2 \cdot 0$ & $1 \cdot 3$ & $1 \cdot 1$ & $0 \cdot 7$ & $2 \cdot 7$ & $1 \cdot 1$ \\
\hline \multirow{2}{*}{\multicolumn{13}{|c|}{ Vitamin $B_{6}(\mathrm{mg})$}} \\
\hline & & & & & & & & & & & & \\
\hline EAR & 0.4 & $0 \cdot 4$ & 0.5 & 0.5 & $0 \cdot 8$ & $0 \cdot 8$ & $1 \cdot 1$ & $1 \cdot 0$ & $1 \cdot 1$ & $1 \cdot 1$ & $1 \cdot 4$ & $1 \cdot 4$ \\
\hline Mean & $1 \cdot 0$ & $1 \cdot 2$ & $1 \cdot 4$ & $1 \cdot 4$ & $1 \cdot 6$ & $1 \cdot 2$ & $1 \cdot 8$ & $1 \cdot 3$ & $1 \cdot 7$ & $1 \cdot 2$ & $1 \cdot 8$ & $1 \cdot 2$ \\
\hline SE & $0 \cdot 3$ & $0 \cdot 1$ & $0 \cdot 1$ & $0 \cdot 1$ & $0 \cdot 1$ & $0 \cdot 1$ & $0 \cdot 1$ & $0 \cdot 1$ & $0 \cdot 1$ & $0 \cdot 0$ & $0 \cdot 2$ & $0 \cdot 1$ \\
\hline$\%$ exceed & $21 \cdot 7$ & $26 \cdot 4$ & $84 \cdot 3$ & $83 \cdot 6$ & $72 \cdot 0$ & $67 \cdot 3$ & $67 \cdot 8$ & $52 \cdot 0$ & $73 \cdot 4$ & $47 \cdot 4$ & $55 \cdot 6$ & $35 \cdot 9$ \\
\hline \multicolumn{13}{|c|}{ Vitamin $B_{12}(\mu \mathrm{g})$} \\
\hline EAR & $0 \cdot 7$ & 0.7 & $1 \cdot 0$ & $1 \cdot 0$ & $1 \cdot 5$ & $1 \cdot 5$ & $2 \cdot 0$ & $2 \cdot 0$ & $2 \cdot 0$ & $2 \cdot 0$ & $2 \cdot 0$ & $2 \cdot 0$ \\
\hline Mean & $2 \cdot 5$ & $2 \cdot 4$ & $3 \cdot 2$ & $3 \cdot 6$ & $3 \cdot 7$ & $3 \cdot 3$ & $3 \cdot 7$ & $2 \cdot 2$ & $4 \cdot 4$ & $2 \cdot 7$ & $5 \cdot 3$ & $2 \cdot 9$ \\
\hline SE & $0 \cdot 3$ & $0 \cdot 4$ & 0.3 & 0.4 & 0.4 & $0 \cdot 8$ & 0.4 & $0 \cdot 2$ & $0 \cdot 3$ & $0 \cdot 3$ & 0.9 & $0 \cdot 3$ \\
\hline$\%$ exceed & $28 \cdot 1$ & $23 \cdot 1$ & $81 \cdot 8$ & $79 \cdot 1$ & $82 \cdot 0$ & $61 \cdot 7$ & $70 \cdot 0$ & $40 \cdot 6$ & $79 \cdot 2$ & $41 \cdot 2$ & $71 \cdot 9$ & $60 \cdot 6$ \\
\hline \multicolumn{13}{|l|}{ Folate $(\mu \mathrm{g})$} \\
\hline EAR & 120 & 120 & 160 & 160 & 250 & 250 & 330 & 330 & 320 & 320 & 320 & 320 \\
\hline Mean & $117 \cdot 0$ & $146 \cdot 3$ & $178 \cdot 0$ & $177 \cdot 4$ & $224 \cdot 8$ & $195 \cdot 5$ & $249 \cdot 0$ & $175 \cdot 1$ & $256 \cdot 1$ & $209 \cdot 0$ & $244 \cdot 0$ & $188 \cdot 4$ \\
\hline SE & $18 \cdot 2$ & $18 \cdot 9$ & $10 \cdot 2$ & $14 \cdot 4$ & $23 \cdot 1$ & $12 \cdot 3$ & $18 \cdot 8$ & $15 \cdot 6$ & $10 \cdot 7$ & $8 \cdot 4$ & $14 \cdot 6$ & $13 \cdot 3$ \\
\hline$\%$ exceed & $11 \cdot 2$ & $13 \cdot 5$ & $45 \cdot 1$ & $48 \cdot 7$ & $34 \cdot 9$ & $23 \cdot 9$ & $24 \cdot 7$ & $10 \cdot 5$ & $26 \cdot 6$ & $14 \cdot 6$ & $20 \cdot 6$ & $12 \cdot 4$ \\
\hline \multicolumn{13}{|c|}{ Vitamin C (mg) } \\
\hline EAR & 13 & 13 & 22 & 22 & 39 & 39 & 63 & 56 & 75 & 60 & 75 & 60 \\
\hline Mean & $72 \cdot 7$ & $59 \cdot 5$ & $87 \cdot 6$ & $89 \cdot 2$ & $94 \cdot 2$ & $101 \cdot 5$ & $124 \cdot 7$ & $108 \cdot 5$ & $104 \cdot 5$ & $82 \cdot 9$ & $86 \cdot 3$ & $75 \cdot 8$ \\
\hline SE & $17 \cdot 7$ & $12 \cdot 1$ & $8 \cdot 2$ & $9 \cdot 1$ & $8 \cdot 2$ & $12 \cdot 5$ & $22 \cdot 1$ & $12 \cdot 5$ & $11 \cdot 3$ & $6 \cdot 0$ & $7 \cdot 6$ & $8 \cdot 5$ \\
\hline$\%$ exceed & $23 \cdot 2$ & $18 \cdot 7$ & $74 \cdot 9$ & $86 \cdot 9$ & $64 \cdot 2$ & $63 \cdot 8$ & $59 \cdot 1$ & $54 \cdot 2$ & $42 \cdot 9$ & $45 \cdot 4$ & $40 \cdot 3$ & $37 \cdot 6$ \\
\hline \multicolumn{13}{|c|}{ Vitamin D (IU)† } \\
\hline EAR & 400 & 400 & 400 & 400 & 400 & 400 & 400 & 400 & 400 & 400 & 400 & 400 \\
\hline Mean & $116 \cdot 7$ & $76 \cdot 3$ & $72 \cdot 4$ & $91 \cdot 3$ & $75 \cdot 3$ & $49 \cdot 5$ & $67 \cdot 7$ & $40 \cdot 0$ & $68 \cdot 4$ & $72 \cdot 4$ & 94.5 & $77 \cdot 3$ \\
\hline SE & $31 \cdot 0$ & $17 \cdot 2$ & $8 \cdot 3$ & $12 \cdot 4$ & $8 \cdot 0$ & $7 \cdot 2$ & $15 \cdot 9$ & $6 \cdot 7$ & $5 \cdot 9$ & $16 \cdot 0$ & $9 \cdot 4$ & $9 \cdot 0$ \\
\hline$\%$ exceed & $1 \cdot 3$ & $2 \cdot 4$ & 0 & 0 & $0 \cdot 7$ & 0 & $2 \cdot 4$ & 0 & 0.3 & $1 \cdot 6$ & $1 \cdot 2$ & $1 \cdot 6$ \\
\hline
\end{tabular}

RE, retinol equivalents; EAR, Estimated Average Requirement.

tTo convert to $\mu \mathrm{g}$, divide IU by 40 .

with the reported increased consumption of fast foods and French fries among children and the high Na content shown in the nutrient composition data of dishes commonly consumed in the Arab Gulf countries ${ }^{(52)}$.

Despite their increased energy intake, more than $90 \%$ of Kuwaiti adults and children did not consume $100 \%$ or more of the EAR for vitamin D, vitamin E, $n-3$ and $n-6$ fatty acids; more than two-thirds did not meet the EAR for $\mathrm{Ca}, \mathrm{Mg}$, folate and fibre; and half did not meet the EAR for vitamins $\mathrm{A}$ and $\mathrm{C}$ and $\mathrm{Zn}$. This indicates low-nutrient-dense food choices. The low intakes of $\mathrm{Ca}, \mathrm{Zn}$, vitamin $\mathrm{D}$ and folate found in the present study are consistent with findings from college women in Kuwait ${ }^{(53)}$ and from adolescents in Saudi Arabia ${ }^{(54,55)}$ and Qatar ${ }^{(56)}$. Moreover, increased prevalence of osteoporosis has been reported in Kuwait ${ }^{(57)}$ and Saudi Arabia ${ }^{(55,58)}$. The US National Health and Nutrition Examination Survey 2001-2002 report identified vitamins $\mathrm{A}, \mathrm{E}$ and $\mathrm{C}$ as potential problems for most gender/age groups based on comparisons with EAR values ${ }^{(59)}$. Vitamin $\mathrm{B}_{6}$ was considered a potential 
Table 5 Weighted means, standard error and percentage consumption of minerals in excess of the Dietary Reference Intakes by age group and gender: nationally representative sample of Kuwaitis, 2009-2010

\begin{tabular}{|c|c|c|c|c|c|c|c|c|c|c|c|c|}
\hline \multirow{2}{*}{$\begin{array}{l}\text { Age (years) } \\
\text { Gender }\end{array}$} & \multicolumn{2}{|c|}{$1-3$} & \multicolumn{2}{|c|}{$4-8$} & \multicolumn{2}{|c|}{$9-13$} & \multicolumn{2}{|c|}{$14-18$} & \multicolumn{2}{|c|}{$19-50$} & \multicolumn{2}{|c|}{$\geq 51$} \\
\hline & Male & Female & Male & Female & Male & Female & Male & Female & Male & Female & Male & Female \\
\hline \multicolumn{13}{|l|}{$\mathrm{Ca}(\mathrm{mg})$} \\
\hline EARt & 500 & 500 & 800 & 800 & 1100 & 1100 & 1100 & 1100 & 800 & $800-1000$ & 1000 & 1000 \\
\hline Mean & 620 & $449 \cdot 3$ & $583 \cdot 9$ & $570 \cdot 6$ & $717 \cdot 6$ & $543 \cdot 8$ & $775 \cdot 3$ & $518 \cdot 7$ & $799 \cdot 7$ & $618 \cdot 4$ & $760 \cdot 2$ & $626 \cdot 4$ \\
\hline SE & $84 \cdot 8$ & $57 \cdot 7$ & $31 \cdot 4$ & $43 \cdot 6$ & $39 \cdot 4$ & $38 \cdot 8$ & $60 \cdot 9$ & $42 \cdot 2$ & $37 \cdot 7$ & $30 \cdot 3$ & $46 \cdot 6$ & $30 \cdot 6$ \\
\hline$\%$ exceed & $14 \cdot 5$ & $8 \cdot 7$ & $20 \cdot 3$ & $19 \cdot 4$ & $16 \cdot 4$ & $6 \cdot 7$ & $21 \cdot 3$ & $6 \cdot 3$ & $41 \cdot 5$ & $23 \cdot 9$ & $4 \cdot 2$ & $10 \cdot 0$ \\
\hline \multicolumn{13}{|l|}{$\mathrm{Fe}(\mathrm{mg})$} \\
\hline EAR & $3 \cdot 0$ & $3 \cdot 0$ & $4 \cdot 1$ & $4 \cdot 1$ & $5 \cdot 9$ & $5 \cdot 7$ & $7 \cdot 7$ & $7 \cdot 9$ & $6 \cdot 0$ & $8 \cdot 1$ & $6 \cdot 0$ & $5 \cdot 0$ \\
\hline Mean & $7 \cdot 8$ & $9 \cdot 9$ & $12 \cdot 2$ & $11 \cdot 4$ & $15 \cdot 2$ & $13 \cdot 2$ & $16 \cdot 9$ & $11 \cdot 7$ & $18 \cdot 2$ & $12 \cdot 4$ & $16 \cdot 5$ & $11 \cdot 3$ \\
\hline SE & $1 \cdot 2$ & $0 \cdot 8$ & $0 \cdot 7$ & $0 \cdot 7$ & $1 \cdot 1$ & $0 \cdot 8$ & $0 \cdot 8$ & 0.7 & 0.6 & 0.4 & 0.9 & $0 \cdot 6$ \\
\hline$\%$ exceed & $24 \cdot 7$ & $27 \cdot 7$ & $92 \cdot 9$ & $93 \cdot 5$ & $87 \cdot 4$ & $90 \cdot 0$ & $92 \cdot 2$ & $69 \cdot 1$ & $96 \cdot 4$ & $71 \cdot 5$ & $92 \cdot 3$ & $93 \cdot 1$ \\
\hline \multicolumn{13}{|l|}{$\mathrm{Mg}(\mathrm{mg})$} \\
\hline EAR & 65 & 65 & 110 & 110 & 200 & 200 & 340 & 300 & 330 & 255 & 350 & 265 \\
\hline Mean & $137 \cdot 2$ & $125 \cdot 2$ & $171 \cdot 8$ & $160 \cdot 3$ & $216 \cdot 6$ & $182 \cdot 1$ & $246 \cdot 0$ & $171 \cdot 6$ & $295 \cdot 4$ & $219 \cdot 8$ & $296 \cdot 7$ & $227 \cdot 4$ \\
\hline SE & 13 & $8 \cdot 4$ & $7 \cdot 9$ & $6 \cdot 6$ & $15 \cdot 7$ & $8 \cdot 7$ & $14 \cdot 1$ & $8 \cdot 5$ & $10 \cdot 5$ & $7 \cdot 9$ & $11 \cdot 8$ & $9 \cdot 6$ \\
\hline$\%$ exceed & $28 \cdot 1$ & $26 \cdot 4$ & $80 \cdot 5$ & $78 \cdot 1$ & $48 \cdot 2$ & $31 \cdot 4$ & $14 \cdot 8$ & $8 \cdot 6$ & $32 \cdot 0$ & $29 \cdot 3$ & $28 \cdot 4$ & $31 \cdot 3$ \\
\hline \multicolumn{13}{|l|}{ P (mg) } \\
\hline EAR & 380 & 380 & 405 & 405 & 1055 & 1055 & 1055 & 1055 & 580 & 580 & 580 & 580 \\
\hline Mean & $709 \cdot 5$ & $578 \cdot 1$ & $876 \cdot 1$ & $785 \cdot 6$ & $1070 \cdot 9$ & $827 \cdot 2$ & $1146 \cdot 0$ & $768 \cdot 6$ & $1241 \cdot 7$ & $863 \cdot 2$ & $1139 \cdot 8$ & $882 \cdot 8$ \\
\hline SE & 83 & $60 \cdot 3$ & $48 \cdot 1$ & $41 \cdot 8$ & $75 \cdot 2$ & $41 \cdot 4$ & $67 \cdot 7$ & $40 \cdot 8$ & $36 \cdot 9$ & $30 \cdot 0$ & $49 \cdot 7$ & $45 \cdot 2$ \\
\hline$\%$ exceed & $27 \cdot 1$ & $21 \cdot 4$ & $87 \cdot 7$ & $81 \cdot 1$ & $41 \cdot 4$ & $27 \cdot 1$ & $51 \cdot 6$ & $14 \cdot 1$ & $88 \cdot 9$ & $74 \cdot 6$ & 84 & $77 \cdot 4$ \\
\hline \multicolumn{13}{|l|}{$\operatorname{Se}(\mu \mathrm{g})$} \\
\hline EAR & 17 & 17 & 23 & 23 & 35 & 35 & 45 & 45 & 45 & 45 & 45 & 45 \\
\hline Mean & $39 \cdot 1$ & $42 \cdot 2$ & $68 \cdot 8$ & $59 \cdot 3$ & 83.5 & $67 \cdot 8$ & $92 \cdot 2$ & $57 \cdot 5$ & $108 \cdot 3$ & $81 \cdot 1$ & $112 \cdot 4$ & $83 \cdot 1$ \\
\hline SE & $5 \cdot 6$ & $9 \cdot 0$ & $5 \cdot 2$ & $5 \cdot 8$ & $8 \cdot 6$ & $4 \cdot 6$ & $5 \cdot 7$ & $4 \cdot 3$ & $4 \cdot 1$ & $6 \cdot 7$ & $6 \cdot 9$ & $5 \cdot 9$ \\
\hline$\%$ exceed & $22 \cdot 2$ & $25 \cdot 2$ & $87 \cdot 0$ & $84 \cdot 5$ & $75 \cdot 5$ & $80 \cdot 7$ & $81 \cdot 5$ & $56 \cdot 9$ & $84 \cdot 7$ & $68 \cdot 8$ & $79 \cdot 1$ & $76 \cdot 3$ \\
\hline \multicolumn{13}{|l|}{$\mathrm{Na}(\mathrm{mg}) \ddagger$} \\
\hline UL & 1500 & 1500 & 1900 & 1900 & 2200 & 2200 & 2300 & 2300 & 2300 & 2300 & 2300 & 2300 \\
\hline Mean & $1607 \cdot 6$ & $2038 \cdot 5$ & $2610 \cdot 7$ & $2339 \cdot 8$ & $3508 \cdot 2$ & $2975 \cdot 7$ & $3679 \cdot 3$ & $2652 \cdot 6$ & $3950 \cdot 3$ & $2857 \cdot 7$ & $3083 \cdot 4$ & $2353 \cdot 8$ \\
\hline SE & $153 \cdot 3$ & $203 \cdot 9$ & $118 \cdot 7$ & $98 \cdot 5$ & $172 \cdot 4$ & $175 \cdot 7$ & $184 \cdot 8$ & 140 & $130 \cdot 4$ & $105 \cdot 3$ & $153 \cdot 4$ & $103 \cdot 4$ \\
\hline$\%$ exceed & $11 \cdot 9$ & $21 \cdot 9$ & $70 \cdot 8$ & $63 \cdot 8$ & $72 \cdot 8$ & $61 \cdot 2$ & $80 \cdot 0$ & $46 \cdot 0$ & $82 \cdot 2$ & $57 \cdot 9$ & $67 \cdot 2$ & $43 \cdot 9$ \\
\hline \multicolumn{13}{|l|}{$\mathrm{Zn}(\mathrm{mg})$} \\
\hline EAR & $2 \cdot 5$ & $2 \cdot 5$ & $4 \cdot 0$ & $4 \cdot 0$ & $7 \cdot 0$ & $7 \cdot 0$ & $8 \cdot 5$ & $7 \cdot 3$ & $9 \cdot 4$ & $6 \cdot 8$ & $9 \cdot 4$ & $6 \cdot 8$ \\
\hline Mean & $5 \cdot 1$ & $5 \cdot 3$ & $7 \cdot 9$ & $7 \cdot 2$ & $9 \cdot 1$ & $7 \cdot 3$ & $10 \cdot 4$ & $6 \cdot 8$ & $11 \cdot 5$ & $7 \cdot 2$ & $9 \cdot 9$ & $7 \cdot 1$ \\
\hline SE & 0.5 & 0.9 & 0.5 & 0.5 & 0.7 & $0 \cdot 4$ & 0.7 & 0.4 & 0.5 & 0.2 & 0.6 & 0.4 \\
\hline$\%$ exceed & $28 \cdot 1$ & $25 \cdot 4$ & $78 \cdot 5$ & $72 \cdot 9$ & $59 \cdot 5$ & $44 \cdot 3$ & $64 \cdot 3$ & $39 \cdot 0$ & $58 \cdot 2$ & $43 \cdot 5$ & $44 \cdot 5$ & $42 \cdot 7$ \\
\hline
\end{tabular}

EAR, Estimated Average Requirement; UL, Tolerable Upper Intake Level.

tThe EAR for $\mathrm{Ca}$ is $800 \mathrm{mg}$ for women aged $19-30$ years and $1000 \mathrm{mg}$ for women aged $31-50$ years.

tThe UL for Na was used to estimate the percentage of participants exceeding the safe Na intake level.

problem for older adult females, $\mathrm{Zn}$ for older adult males and females and teenage females, and $\mathrm{P}$ for pre-teens and teenage females. These findings are comparable to the results of the current study.

Unlike a previous report showing low Fe intake among anaemic and non-anaemic girls aged 14-20 years in Kuwait $(10.6 \text { and } 10 \cdot 8 \mathrm{mg} / \mathrm{d} \text {, respectively })^{(60)}$, the current study showed a mean Fe intake of $11.7 \mathrm{mg} / \mathrm{d}$ for the same gender and age group.

Similar to our findings, the British Columbia Nutrition Survey reported that income level did not affect nutrient intakes while educational attainment affected only the intakes of vitamins $\mathrm{C}$ and $\mathrm{B}_{12}{ }^{(61)}$. On the contrary, the trend in nutrient intake among Mexican Americans between 1982 and 2006 showed increased energy and fat intakes and decreased total protein and carbohydrate intakes with increasing income and educational attainment ${ }^{(62)}$. The lack of effect of income on food intake among Kuwaitis could be explained by low food prices and current subsidy policies. All Kuwaiti citizens are eligible to subsidized food. Subsidized food items include polished white rice, refined table sugar, lentils, tomato paste, powdered full-fat milk or long-life liquid full-fat or skimmed milk, infant formula, instant cereal, vegetable oil, frozen chicken and full-fat spread cheeses. The current Kuwaiti consumption patterns highlight the importance of revisiting subsidy policies to gear them towards subsidizing healthy foods. There is a need to substitute energy-dense foods (oil, sugar, ghee) with more nutrient-rich, Ca-rich fruits and vegetables.

\section{Limitations}

Estimation bias of the dietary measurements cannot be excluded because of the present study's reliance on the assessment of a single $24 \mathrm{~h}$ recall. Multiple $24 \mathrm{~h}$ recalls would be necessary to confirm individual intakes. Privacy of held food and cultural beliefs was a real challenge to dietary data collection and limited our ability to collect a second $24 \mathrm{~h}$ recall even for a subsample. However, the results of biochemical tests revealed that the majority of 
Table 6 Unweighted mean macronutrient intakes of adults according to demographic characteristics: nationally representative sample of Kuwaitis, 2009-2010

\begin{tabular}{|c|c|c|c|c|c|c|c|}
\hline Characteristic & Energy (kJ) & Energy (kcal) & Carbohydrates (g) & Protein $(\mathrm{g})$ & Fat $(\mathrm{g})$ & Cholesterol (mg) & Fibre (g) \\
\hline \multicolumn{8}{|l|}{ Gender } \\
\hline Male & 9829 & $2349 \cdot 1$ & $312 \cdot 2$ & $101 \cdot 9$ & $79 \cdot 8$ & $305 \cdot 2$ & $27 \cdot 1$ \\
\hline Female & 6797 & $1624 \cdot 5$ & $218 \cdot 6$ & $66 \cdot 5$ & $56 \cdot 1$ & $202 \cdot 1$ & $20 \cdot 4$ \\
\hline$P$ & $0 \cdot 0$ & $0 \cdot 0$ & $0 \cdot 0$ & $0 \cdot 0$ & $0 \cdot 0$ & 0.0 & 0.0 \\
\hline \multicolumn{8}{|l|}{ Marital status } \\
\hline Married & 8274 & $1977 \cdot 5$ & $265 \cdot 0$ & $83 \cdot 8$ & $67 \cdot 3$ & $250 \cdot 4$ & $24 \cdot 4$ \\
\hline Not married & 8525 & $2037 \cdot 4$ & $267 \cdot 5$ & $84 \cdot 6$ & $72 \cdot 1$ & $263 \cdot 4$ & $21 \cdot 5$ \\
\hline Widowed & 6004 & $1434 \cdot 9$ & $191 \cdot 5$ & $65 \cdot 7$ & $47 \cdot 2$ & $201 \cdot 3$ & $18 \cdot 8$ \\
\hline Divorced & 7073 & $1690 \cdot 4$ & $236 \cdot 0$ & $66 \cdot 4$ & $56 \cdot 0$ & $202 \cdot 8$ & $18 \cdot 2$ \\
\hline$P$ & 0.0 & 0.0 & 0.0 & 0.004 & 0.0 & $0 \cdot 154$ & 0.008 \\
\hline \multicolumn{8}{|l|}{ Education } \\
\hline Illiterate/functional illiterate & 6091 & $1455 \cdot 9$ & $208 \cdot 4$ & $66 \cdot 0$ & $42 \cdot 1$ & $182 \cdot 7$ & $18 \cdot 6$ \\
\hline$<$ High school & 8389 & $2005 \cdot 0$ & $274 \cdot 5$ & $87 \cdot 8$ & $64 \cdot 5$ & $267 \cdot 8$ & $24 \cdot 5$ \\
\hline High school & 7968 & $1904 \cdot 5$ & $250 \cdot 3$ & 78.9 & $67 \cdot 5$ & $246 \cdot 8$ & $22 \cdot 2$ \\
\hline$>$ High school & 8768 & $2095 \cdot 5$ & $277 \cdot 6$ & $87 \cdot 1$ & $73 \cdot 5$ & $251 \cdot 6$ & $25 \cdot 5$ \\
\hline$P$ & 0.0 & 0.0 & 0.0 & 0.0 & 0.0 & 0.027 & 0.004 \\
\hline \multicolumn{8}{|l|}{ Occupation } \\
\hline Self-employed & 9611 & $2297 \cdot 0$ & $300 \cdot 2$ & $109 \cdot 3$ & $76 \cdot 8$ & $272 \cdot 8$ & $36 \cdot 7$ \\
\hline Salary employed & 8917 & $2131 \cdot 1$ & $280 \cdot 3$ & $88 \cdot 4$ & $75 \cdot 3$ & $266 \cdot 1$ & $23 \cdot 4$ \\
\hline Retired & 8271 & $1976 \cdot 8$ & $274 \cdot 1$ & $88 \cdot 0$ & $61 \cdot 9$ & $253 \cdot 5$ & $28 \cdot 0$ \\
\hline Full-time work at home & 6626 & $1583 \cdot 7$ & $215 \cdot 3$ & $67 \cdot 5$ & $52 \cdot 7$ & $214 \cdot 7$ & $21 \cdot 5$ \\
\hline Unemployed & 8019 & $1916 \cdot 6$ & $249 \cdot 3$ & $76 \cdot 8$ & $70 \cdot 1$ & $236 \cdot 6$ & $17 \cdot 4$ \\
\hline$P$ & 0.0 & 0.0 & $0 \cdot 0$ & 0.0 & $0 \cdot 0$ & 0.046 & $0 \cdot 0$ \\
\hline \multicolumn{8}{|l|}{ Monthly family income (KD) } \\
\hline$<1000$ & 8071 & $1929 \cdot 0$ & $259 \cdot 2$ & $81 \cdot 9$ & $65 \cdot 4$ & $239 \cdot 9$ & $22 \cdot 8$ \\
\hline 1000-1499 & 8005 & $1913 \cdot 2$ & $256 \cdot 5$ & $80 \cdot 3$ & $65 \cdot 6$ & $233 \cdot 4$ & $22 \cdot 6$ \\
\hline 1500-1999 & 8597 & $2054 \cdot 7$ & $275 \cdot 8$ & $86 \cdot 9$ & $69 \cdot 6$ & $255 \cdot 8$ & $24 \cdot 8$ \\
\hline$>2000$ & 7932 & $1895 \cdot 9$ & $250 \cdot 1$ & $80 \cdot 2$ & $66 \cdot 0$ & $265 \cdot 2$ & $23 \cdot 3$ \\
\hline$P$ & $0 \cdot 179$ & $0 \cdot 179$ & $0 \cdot 109$ & 0.298 & $0 \cdot 618$ & 0.311 & 0.465 \\
\hline
\end{tabular}

KD, Kuwaiti Dinars.

$P<0.05$.

Table 7 Unweighted mean vitamin intakes of adults according to demographic characteristics: nationally representative sample of Kuwaitis, 2009-2010

\begin{tabular}{|c|c|c|c|c|c|c|c|c|c|c|}
\hline Characteristic & $\begin{array}{l}\text { Thiamin } \\
(\mathrm{mg})\end{array}$ & $\begin{array}{l}\text { Riboflavin } \\
\text { (mg) }\end{array}$ & $\begin{array}{l}\text { Niacin } \\
(\mathrm{mg})\end{array}$ & $\begin{array}{l}\text { Vitamin } B_{6} \\
(\mathrm{mg})\end{array}$ & $\begin{array}{l}\text { Vitamin } \\
\mathrm{B}_{12}(\mu \mathrm{g})\end{array}$ & $\begin{array}{l}\text { Folate } \\
(\mu \mathrm{g})\end{array}$ & $\begin{array}{l}\text { Pantothenic } \\
\text { acid (mg) }\end{array}$ & $\begin{array}{l}\text { Vitamin } C \\
(\mathrm{mg})\end{array}$ & $\begin{array}{l}\text { Vitamin } E \\
(\mathrm{mg})\end{array}$ & $\begin{array}{c}\text { Vitamin } A \\
\text { ( } \mu \mathrm{g} R E)\end{array}$ \\
\hline \multicolumn{11}{|l|}{ Gender } \\
\hline Male & $3 \cdot 1$ & $1 \cdot 8$ & $24 \cdot 3$ & $1 \cdot 7$ & $4 \cdot 2$ & $261 \cdot 6$ & $5 \cdot 0$ & $97 \cdot 2$ & $5 \cdot 6$ & $1019 \cdot 1$ \\
\hline Female & 1.5 & $1 \cdot 3$ & $16 \cdot 3$ & $1 \cdot 2$ & $2 \cdot 8$ & $199 \cdot 6$ & $3 \cdot 4$ & $83 \cdot 3$ & $4 \cdot 6$ & $1110 \cdot 7$ \\
\hline$P$ & 0.0 & 0.0 & $0 \cdot 0$ & 0.0 & 0.0 & 0.0 & 0.0 & 0.024 & 0.0 & 0.429 \\
\hline \multicolumn{11}{|l|}{ Marital status } \\
\hline Married & $2 \cdot 3$ & $1 \cdot 6$ & $20 \cdot 0$ & $1 \cdot 4$ & 3.5 & $233 \cdot 0$ & $4 \cdot 3$ & $89 \cdot 6$ & $5 \cdot 2$ & $1131 \cdot 3$ \\
\hline Not married & $1 \cdot 8$ & $1 \cdot 4$ & $21 \cdot 0$ & $1 \cdot 4$ & $3 \cdot 5$ & $227 \cdot 2$ & $4 \cdot 0$ & $104 \cdot 5$ & $5 \cdot 1$ & $885 \cdot 7$ \\
\hline Widowed & $1 \cdot 3$ & $1 \cdot 2$ & $16 \cdot 4$ & $1 \cdot 1$ & $2 \cdot 8$ & $174 \cdot 5$ & $3 \cdot 3$ & $62 \cdot 0$ & 3.9 & $1140 \cdot 0$ \\
\hline Divorced & 1.9 & $1 \cdot 3$ & $17 \cdot 1$ & $1 \cdot 2$ & $2 \cdot 8$ & $189 \cdot 0$ & $3 \cdot 2$ & $60 \cdot 8$ & $4 \cdot 3$ & $606 \cdot 3$ \\
\hline$P$ & $0 \cdot 136$ & 0.011 & 0.073 & 0.046 & 0.52 & 0.025 & 0.004 & 0.008 & $0 \cdot 194$ & $0 \cdot 158$ \\
\hline \multicolumn{11}{|l|}{ Education } \\
\hline $\begin{array}{l}\text { Illiterate/functional } \\
\text { illiterate }\end{array}$ & $2 \cdot 3$ & $1 \cdot 3$ & $14 \cdot 8$ & $1 \cdot 1$ & $3 \cdot 1$ & $154 \cdot 8$ & $3 \cdot 7$ & $46 \cdot 2$ & $3 \cdot 2$ & $550 \cdot 6$ \\
\hline$<$ High school & $2 \cdot 5$ & $1 \cdot 6$ & $21 \cdot 2$ & 1.5 & 3.9 & $229 \cdot 1$ & $4 \cdot 7$ & $90 \cdot 1$ & 4.9 & $926 \cdot 3$ \\
\hline High school & $2 \cdot 1$ & $1 \cdot 4$ & $19 \cdot 3$ & $1 \cdot 3$ & $3 \cdot 3$ & $221 \cdot 5$ & $3 \cdot 8$ & $88 \cdot 2$ & 4.9 & $1129 \cdot 4$ \\
\hline$>$ High school & $2 \cdot 0$ & $1 \cdot 6$ & $20 \cdot 9$ & 1.5 & $3 \cdot 4$ & $253 \cdot 1$ & $4 \cdot 1$ & $102 \cdot 3$ & $5 \cdot 8$ & $1233 \cdot 5$ \\
\hline$P$ & 0.525 & 0.006 & 0.001 & 0.0 & 0.331 & 0.0 & $0 \cdot 0$ & 0.0 & $0 \cdot 0$ & 0.017 \\
\hline \multicolumn{11}{|l|}{ Occupation } \\
\hline Self-employed & $2 \cdot 2$ & $1 \cdot 5$ & $23 \cdot 4$ & 1.9 & $2 \cdot 5$ & $313 \cdot 6$ & $5 \cdot 7$ & $120 \cdot 9$ & $5 \cdot 9$ & $1021 \cdot 2$ \\
\hline Salary employed & $2 \cdot 4$ & $1 \cdot 6$ & $21 \cdot 9$ & $1 \cdot 4$ & 3.5 & $239 \cdot 8$ & $4 \cdot 2$ & $96 \cdot 1$ & $5 \cdot 3$ & $934 \cdot 7$ \\
\hline Retired & $2 \cdot 7$ & $1 \cdot 7$ & $20 \cdot 6$ & 1.5 & $4 \cdot 1$ & $237 \cdot 5$ & $4 \cdot 7$ & $85 \cdot 4$ & $5 \cdot 1$ & $1359 \cdot 4$ \\
\hline Full-time work at home & $1 \cdot 7$ & $1 \cdot 4$ & $15 \cdot 9$ & $1 \cdot 2$ & $3 \cdot 1$ & $200 \cdot 7$ & $3 \cdot 7$ & $78 \cdot 5$ & $4 \cdot 7$ & $1236 \cdot 7$ \\
\hline Unemployed & 1.5 & $1 \cdot 2$ & $18 \cdot 4$ & $1 \cdot 3$ & $3 \cdot 0$ & $205 \cdot 9$ & $3 \cdot 3$ & $91 \cdot 8$ & $4 \cdot 6$ & $712 \cdot 4$ \\
\hline$P$ & 0.031 & $0 \cdot 0$ & $0 \cdot 0$ & 0.0 & $0 \cdot 131$ & 0.002 & $0 \cdot 0$ & $0 \cdot 149$ & 0.317 & 0.008 \\
\hline \multicolumn{11}{|l|}{$\begin{array}{l}\text { Monthly family income } \\
\text { (KD) }\end{array}$} \\
\hline$<1000$ & $2 \cdot 2$ & $1 \cdot 4$ & $20 \cdot 5$ & $1 \cdot 4$ & $3 \cdot 3$ & $221 \cdot 3$ & $4 \cdot 0$ & $82 \cdot 9$ & $5 \cdot 0$ & $866 \cdot 0$ \\
\hline $1000-1499$ & $2 \cdot 1$ & 1.5 & $18 \cdot 9$ & $1 \cdot 3$ & $3 \cdot 4$ & $220 \cdot 8$ & $4 \cdot 0$ & $88 \cdot 0$ & $5 \cdot 0$ & $1032 \cdot 4$ \\
\hline 1500-1999 & $2 \cdot 2$ & $1 \cdot 7$ & $21 \cdot 9$ & 1.5 & $3 \cdot 7$ & $232 \cdot 5$ & 4.5 & $91 \cdot 4$ & $5 \cdot 0$ & $1090 \cdot 8$ \\
\hline$>2000$ & $2 \cdot 1$ & $1 \cdot 5$ & $18 \cdot 1$ & $1 \cdot 4$ & $3 \cdot 4$ & $235 \cdot 0$ & $4 \cdot 0$ & $95 \cdot 8$ & $5 \cdot 1$ & $1287 \cdot 0$ \\
\hline$P$ & 0.993 & 0.04 & 0.005 & 0.471 & 0.779 & 0.635 & 0.074 & 0.519 & 0.984 & 0.09 \\
\hline
\end{tabular}

$\mathrm{RE}$, retinol equivalents; KD, Kuwaiti Dinars.

$P<0.05$. 
Table 8 Unweighted mean mineral intakes of adults according to demographic characteristics: nationally representative sample of Kuwaitis, 2009-2010

\begin{tabular}{|c|c|c|c|c|c|c|c|}
\hline & $\mathrm{Ca}(\mathrm{mg})$ & $\mathrm{Fe}(\mathrm{mg})$ & $P(m g)$ & $\operatorname{Se}(\mu \mathrm{g})$ & $\mathrm{Na}(\mathrm{mg})$ & $\mathrm{Zn}(\mathrm{mg})$ & $\mathrm{Mg}(\mathrm{mg})$ \\
\hline \multicolumn{8}{|l|}{ Gender } \\
\hline Male & $809 \cdot 4$ & $17 \cdot 7$ & $1215 \cdot 1$ & $110 \cdot 5$ & $3714 \cdot 0$ & $10 \cdot 8$ & $300 \cdot 7$ \\
\hline Female & $616 \cdot 2$ & $12 \cdot 2$ & $857 \cdot 1$ & $77 \cdot 1$ & $2714 \cdot 0$ & $7 \cdot 2$ & $219 \cdot 1$ \\
\hline$P$ & 0.0 & $0 \cdot 0$ & 0.0 & $0 \cdot 0$ & 0.0 & 0.0 & 0.0 \\
\hline \multicolumn{8}{|l|}{ Marital status } \\
\hline Married & $731 \cdot 2$ & $15 \cdot 0$ & $1046 \cdot 7$ & $95 \cdot 0$ & $3201 \cdot 2$ & $9 \cdot 0$ & $266 \cdot 3$ \\
\hline Not married & $638 \cdot 6$ & $14 \cdot 7$ & $976 \cdot 1$ & $84 \cdot 0$ & $3255 \cdot 0$ & $8 \cdot 7$ & $226 \cdot 4$ \\
\hline Widowed & $589 \cdot 4$ & $10 \cdot 9$ & $868 \cdot 4$ & $91 \cdot 5$ & $2514 \cdot 8$ & $6 \cdot 8$ & $230 \cdot 1$ \\
\hline Divorced & $585 \cdot 0$ & $13 \cdot 5$ & $829 \cdot 3$ & $71 \cdot 6$ & $2847 \cdot 1$ & $7 \cdot 1$ & $209 \cdot 6$ \\
\hline$P$ & 0.002 & 0.004 & 0.005 & 0.044 & 0.033 & 0.004 & $0 \cdot 0$ \\
\hline \multicolumn{8}{|l|}{ Education } \\
\hline Illiterate/functional illiterate & $574 \cdot 5$ & $10 \cdot 2$ & $853 \cdot 9$ & $84 \cdot 8$ & $2415 \cdot 4$ & $7 \cdot 1$ & $207 \cdot 2$ \\
\hline$<$ High school & $732 \cdot 1$ & $15 \cdot 2$ & $1100 \cdot 3$ & $102 \cdot 8$ & $3155 \cdot 0$ & $9 \cdot 4$ & $275 \cdot 8$ \\
\hline High school & $667 \cdot 9$ & $14 \cdot 3$ & $960 \cdot 9$ & $84 \cdot 8$ & $3109 \cdot 4$ & $8 \cdot 4$ & $240 \cdot 4$ \\
\hline$>$ High school & $762 \cdot 7$ & $16 \cdot 0$ & $1074 \cdot 7$ & $95 \cdot 8$ & $3435 \cdot 3$ & $9 \cdot 2$ & $273 \cdot 8$ \\
\hline$P$ & 0.001 & $0 \cdot 0$ & 0.0 & 0.004 & 0.0 & 0.002 & $0 \cdot 0$ \\
\hline \multicolumn{8}{|l|}{ Occupation } \\
\hline Self-employed & $826 \cdot 4$ & $20 \cdot 9$ & $1297 \cdot 9$ & $105 \cdot 6$ & $3708 \cdot 1$ & $10 \cdot 8$ & $360 \cdot 8$ \\
\hline Salary employed & $736 \cdot 6$ & $15 \cdot 7$ & $1062 \cdot 0$ & $94 \cdot 8$ & $3399 \cdot 8$ & $9 \cdot 3$ & $264 \cdot 6$ \\
\hline Retired & 775.9 & $15 \cdot 7$ & $1128 \cdot 3$ & $105 \cdot 0$ & $3199 \cdot 3$ & $9 \cdot 6$ & $294 \cdot 4$ \\
\hline Full-time work at home & $625 \cdot 1$ & $12 \cdot 2$ & 891.5 & $84 \cdot 2$ & $2649 \cdot 3$ & $7 \cdot 4$ & $230 \cdot 1$ \\
\hline Unemployed & $582 \cdot 9$ & $13 \cdot 5$ & $871 \cdot 8$ & $71 \cdot 8$ & $3186 \cdot 0$ & $7 \cdot 6$ & $189 \cdot 5$ \\
\hline$P$ & 0.0 & $0 \cdot 0$ & 0.0 & 0.0 & 0.0 & 0.0 & 0.0 \\
\hline \multicolumn{8}{|l|}{ Monthly family income (KD) } \\
\hline$<1000$ & $666 \cdot 8$ & $14 \cdot 7$ & $991 \cdot 4$ & 94.9 & $3129 \cdot 9$ & $8 \cdot 4$ & $254 \cdot 4$ \\
\hline $1000-1499$ & $721 \cdot 5$ & $14 \cdot 2$ & $1018 \cdot 9$ & $92 \cdot 9$ & $3083 \cdot 2$ & $8 \cdot 8$ & $253 \cdot 8$ \\
\hline $1500-1999$ & $709 \cdot 1$ & $15 \cdot 5$ & $1059 \cdot 8$ & $89 \cdot 4$ & 3285.9 & $9 \cdot 3$ & $263 \cdot 4$ \\
\hline$>2000$ & $708 \cdot 7$ & $14 \cdot 4$ & $995 \cdot 1$ & $91 \cdot 0$ & $3149 \cdot 2$ & $8 \cdot 6$ & $250 \cdot 4$ \\
\hline$P$ & 0.491 & 0.273 & 0.441 & 0.804 & 0.611 & 0.272 & 0.733 \\
\hline
\end{tabular}

KD, Kuwaiti Dinars.

$P<0.05$.

participants had all vitamin and trace element levels within the normal range. The detailed blood sample analysis and its association with dietary indices are beyond the scope of the current paper.

\section{Conclusions}

Kuwait is a country with a food-abundant environment. Food is available at low cost and Kuwaitis have low nutrition awareness. In this context Kuwaitis are experiencing a nutrition transition that is evidenced by increased prevalences of obesity, overweight and nutrition-related NCD and high consumption of foods that are energy dense, high in macronutrients and low in fibre and micronutrient density. The present study quantifies food intake in a representative sample of Kuwaiti individuals. As such, it represents a critical step in understanding how to modify dietary intake to reduce the prevalence of nutrition-related NCD in Kuwaitis. There is an urgent need to increase nutrition awareness of healthy food choices and to conduct interventions aimed at modifying subsidy policies in Kuwait and limiting $\mathrm{Na}$ intake.

Further analyses are needed to identify high energy and $\mathrm{Na}$ sources in the Kuwaiti diet and to estimate the differential contributions of Western $v$. traditional foods (such as soft drinks and fruit drinks $v$. milk and fruit juices) to energy and nutrient intakes and to determine whether Kuwaitis are meeting the recommended intakes of fruit and vegetables.

\section{Acknowledgements}

Sources of funding: The study was supported in part by grant \#2003-1202-02 from the Kuwait Foundation for the Advancement of Science; the Kuwait Supreme Council for Planning and Development; and the UN Development Programme. Conflicts of interest: The authors declare they have no conflicts of interest. Authors' contributions: S.Z. conceived of and designed the dietary assessment tools and questionnaires for the specific age groups, developed the dietary assessment database, verified the field survey dietary data collection, conducted the analysis and interpretation of data, and wrote the manuscript. S.N.A.-H. conceived of the study, obtained the funding grant, ensured the provision of materials, analysis tools and consultations needed for dietary and anthropometric measurements, and was responsible for data compilation and interpretation. N.A.-H. participated in the experimental design and ensured the provision of all facilities - including study sites, health clinics and team members from the Ministry of Health - needed for receiving household members, completing the survey questionnaires and taking 
measurements. S.A.-Z. and H.A. recruited and supervised the field survey teams required for recruitment of clusters of households from different localities and line listing of household individuals, and developed the database of recruited households for demographic data and line listing of household members. I.I. and H.A.-A. were responsible for entry and coding of all $24 \mathrm{~h}$ dietary data and dietary data processing and tabulation. A.A.-O. developed and maintained the study database by developing a web-based demographic template for recruited households and a template of age-specific questionnaires for daily upload of completed questionnaires, and assisted in data cleaning and data analysis. E.A.-S. and M.A.-S. were responsible for supervision and setting of criteria for anthropometric data collection and interpretation. R.T.J. reviewed and edited the manuscript. Acknowledgements: The authors express their gratitude to the management of the Kuwait Institute for Scientific Research and the Ministry of Health for support of their scientific pursuit. Thanks are also extended to the funding bodies for their contribution towards the project.

\section{References}

1. World Health Organization (2003) Diet, Nutrition, and the Prevention of Chronic Diseases. Joint WHO/FAO Consultation. WHO Technical Report Series no. 916. Geneva: WHO.

2. Ng SW, Zaghloul S, Ali HI et al. (2011) The prevalence and trends of overweight, obesity and nutrition-related noncommunicable diseases in the Arabian Gulf States. Obes Rev 12, 1-13.

3. Al Rashdan IR \& Nesef YA (2010) Prevalence of overweight, obesity and metabolic syndrome among adult Kuwaitis: results from community-based national survey. Angiology 61, 42-48.

4. Abdella N, Al Arouj M, Al Nakhi A et al. (1998) Noninsulin-dependent diabetes in Kuwait: prevalence rates and associated risk factors. Diabetes Res Clin Pract 42, 187-196.

5. Jackson RT, Al-Moussa Z, Al-Raqua M et al. (2002) Multiple coronary risk factors in healthy older Kuwaiti males. Eur J Clin Nutr 56, 709-714.

6. Jackson RT, Al-Moussa Z, Al-Raqua M et al. (2001) Prevalence of coronary risk factors in healthy adult Kuwaitis. Int J Food Sci Nutr 52, 301-311.

7. Food and Nutrition Administration (editor) (2001-2004) Kuwait Nutrition Surveillance. Kuwait: Ministry of Health.

8. El-Bayoumy I, Shady I \& Lotfy H (2009) The prevalence of obesity among adolescents (10 to 14) in Kuwait. Asia Pac J Public Health 21, 153-159.

9. Food and Agriculture Organization of the United Nations (2006) Kuwait Nutrition Profile. Rome: Nutrition and Consumer Protection Division, FAO; available at $\mathrm{ftp}: / /$ ftp.fao.org/ag/agn/nutrition/ncp/kwt.pdf

10. World Health Organization (2009) World Health Statistics 2009. Table 9: Demographic and socioeconomic statistics. http://www.who.int/whosis/whostat/2009/en/ (accessed September 2011).

11. International Monetary Fund (2011) World Economic Outlook Database. http://www.imf.org/external/pubs/ft/ weo/2011/01/weodata/index.aspx (accessed April 2011).

12. Population Division, Department of Economic and Social Affairs, United Nations Secretariat (2005) World Population Prospects. The 2004 Revision. Highlights. New York: UN; available at http://www.un.org/esa/population/publications/ WPP2004/2004Highlights_finalrevised.pdf
13. Musaiger AO (1990) Nutritional disorders associated with affluence in Bahrain. Fam Pract 7, 9.

14. Popkin BM (2004) The nutrition transition: an overview of world patterns of change. Nutr Rev 62, 7 Pt 2, S140-S143.

15. Popkin BM (2006) Global nutrition dynamics: the world is shifting rapidly toward a diet linked with noncommunicable diseases. Am J Clin Nutr 84, 289-298.

16. Popkin BM (2011) Contemporary nutritional transition: determinants of diet and its impact on body composition. Proc Nutr Soc 70, 82-91.

17. Poskitt EM (2009) Countries in transition: underweight to obesity non-stop? Ann Trop Paediatr 29, 1-11.

18. Piernas C \& Popkin BM (2010) Snacking increased among US adults between 1977 and 2006. J Nutr 140, 325-332.

19. Piernas C \& Popkin BM (2011) Food portion patterns and trends among US children and the relationship to total eating occasion size, 1977-2006. J Nutr 141, 1159-1164.

20. Astrup A, Dyerberg J, Selleck M et al. (2008) Nutrition transition and its relationship to the development of obesity and related chronic diseases. Obes Rev 9, Suppl. 1, 48-52.

21. Zaghloul S, Waslien C, Al Somaie M et al. (2012) Low adherence of Kuwaiti adults to dietary guidelines for daily fruit and vegetable intake. East Mediterr Health $J$ 18, 461-467.

22. Serour M, Alqhenaei H, Al-Saqabi S et al. (2007) Cultural factors and patients' adherence to lifestyle measures. $\mathrm{Br} \mathrm{J}$ Gen Pract 57, 291-295.

23. Honkala S, Honkala E \& Al-Sahli N (2006) Consumption of sugar products and associated life- and school-satisfaction and self-esteem factors among schoolchildren in Kuwait. Acta Odontol Scand 64, 79-88.

24. Al-Ansari JM, Al-Jairan LY \& Gillespie GM (2006) Dietary habits of the primary to secondary school population and implications for oral health. J Allied Health 35, 75-80.

25. World Health Organization (1995) Physical Status: The Use and Interpretation of Anthropometry. Report of a WHO Expert Committee. WHO Technical Report Series no. 854. Geneva: WHO.

26. World Health Organization (2006) WHO Child Growth Standards: Length/Height-for-Age, Weight-for-Age, Weightfor-Height and Body Mass Index-for-Age: Methods and Development. Geneva: WHO; available at http:// www.who.int/childgrowth/publications/technical_report_ $\mathrm{pub} / \mathrm{en} /$ index.html

27. de Onis M, Onyango AW, Borghi E et al. (2007) Development of a WHO growth reference for school-aged children and adolescents. Bull World Health Organ 85, 660-667.

28. Conway JM, lngwersen LA, Vinyard BT et al. (2003) Effectiveness of the US Department of Agriculture 5-step multiple-pass method in assessing food intake in obese and nonobese women. Am J Clin Nutr 77, 1171-1178.

29. Conway JM, Ingwersen LA \& Moshfegh AJ (2004) Accuracy of dietary recall using the USDA five-step multiple-pass method in men: an observational validation study. $J \mathrm{Am}$ Diet Assoc 104, 595-603.

30. Zaghloul S, Ilyan I, Al-Hamly S et al. (2010) Kuwait Food Instruction Booklet. Kuwait: Kuwait Institute for Scientific Research.

31. Schofield WN (1984) Predicting basal metabolic rate, new standards and review of previous work. Hum Nutr Clin Nutr 39C, Suppl. 1, 5-41.

32. Harrison GG, Galal OM, Ibrahim N et al. (2000) Underreporting of food intake by dietary recall is not universal: a comparison of data from Egyptian and American women. J Nutr 130, 2049-2054.

33. Briefel RE, Sempos CT, McDowell MA et al. (1997) Dietary methods research in the third National Health and Nutrition Examination Survey: underreporting of energy intake. $\mathrm{Am} \mathrm{J}$ Clin Nutr 65, 4 Suppl., 1203S-1209S. 
34. Food and Nutrition Board, Institute of Medicine (2001) Dietary Reference Intakes for Energy, Carbohydrate, Fiber, Fat, Fatty Acids, Cholesterol, Protein, and Amino Acids. Washington, DC: National Academy Press.

35. ESHA Research (2006) Food Processor and Genesis SQL Database Sources. Salem, OR: ESHA Research.

36. Al-Amiri HA, Al-Otaibi JA, Al-Foudari MY et al. (2009) The nutritive value of selected commonly consumed Kuwaiti dishes. Int J Food Sci Nutr 60, 668-676.

37. Al-Amiri HA, Al-Hamad NM, Al-Awadhi FA et al. (2011) Total, insoluble and soluble dietary fiber contents of selected Kuwaiti composite dishes. Int J Food Sci Nutr 62, 152-157.

38. Dashti B, Al-Awadi F, Khalafawi MS et al. (2003) Soluble and insoluble dietary fibre in thirty-two Kuwaiti dishes. Food Chem 83, 557-561.

39. Dashti B, Al-Awadi F, Al-Kandari R et al. (2004) Macro and microelements contents of 32 Kuwaiti composite dishes. Food Chem 85, 331-337.

40. Dashti BH, Al-Awadi F, Khalafawi MS et al. (2001) Nutrient contents of some traditional Kuwaiti dishes: proximate composition, and phytate content. Food Chem 74, 169-175.

41. Kuwait Institute for Scientific Research (1998) Food Composition Tables for Kuwait Composite Dishes. Safat: Kuwait Institute for Scientific Research.

42. Food and Nutrition Board, Institute of Medicine (1997) Dietary Reference Intakes for Thiamin, Riboflavin, Niacin, Vitamin $B_{6}$, Folate, Vitamin $B_{12}$, Pantothenic Acid, Biotin, and Choline. Washington, DC: National Academy Press.

43. Food and Nutrition Board, Institute of Medicine (1998) Dietary Reference Intakes for Vitamin C, Vitamin E, Selenium, and Carotenoids. Washington, DC: National Academy Press.

44. Food and Nutrition Board, Institute of Medicine (2000) Dietary Reference Intakes for Vitamin A, Vitamin K, Arsenic, Boron, Chromium, Copper, Iodine, Iron, Manganese, Molybdenum, Nickel, Silicon, Vanadium, and Zinc. Washington, DC: National Academy Press.

45. Food and Nutrition Board, Institute of Medicine (2002) Dietary Reference Intakes for Calcium, Phosphorus, Magnesium, Vitamin D, and Fluoride. Washington, DC: National Academy Press.

46. Food and Nutrition Board, Institute of Medicine (2004) Dietary Reference Intakes for Water, Potassium, Sodium, Chloride, and Sulfate. Washington, DC: National Academy Press.

47. Food and Nutrition Board, Institute of Medicine (2005) Dietary Reference Intakes for Energy, Carbohydrate, Fiber, Fat, Fatty Acids, Cholesterol, Protein, and Amino Acids. Washington, DC: National Academy Press Institute of Medicine.

48. Food and Nutrition Board, Institute of Medicine (2011) Dietary Reference Intakes for Calcium and Vitamin D. Washington, DC: National Academy Press.
49. Sibai AM, Nasreddine L, Mokdad AH et al. (2010) Nutrition transition and cardiovascular risk factors in Middle East and North African countries: reviewing the evidence. Ann Nutr Metab 57, 193-203.

50. Musaiger AO (2011) Food Consumption Patterns in the Eastern Mediterranean Region, 1st ed. Manama-Bahrain: Arab Center for Nutrition.

51. Al-Kandari YY (2006) Prevalence of obesity in Kuwait and its relation to sociocultural variables. Obes Rev 7, 147-154.

52. Musaiger AO, Takruri HR, Hasan AS et al. (2012) Foodbased dietary guidelines for the Arab Gulf countries. J Nutr Metab 2012, article ID 905303; available at http://www.hindawi. com/journals/jnume/2012/905303/

53. Al-Shawi AN (1992) Nutrient intakes of university women in Kuwait. $J$ R Soc Health 112, 114-118.

54. Washi SA \& Ageib MB (2010) Poor diet quality and food habits are related to impaired nutritional status in 13- to 18-year-old adolescents in Jeddah. Nutr Res 30, 527-534.

55. Sadat-Ali M, AlElq A, Al-Turki H et al. (2009) Vitamin D levels in healthy men in eastern Saudi Arabia. Ann Saudi Med 29, 378-382.

56. Bener A, Al-Ali M \& Hoffmann GF (2009) High prevalence of vitamin D deficiency in young children in a highly sunny humid country: a global health problem. Minerva Pediatr 61, 15-22.

57. Dougherty G \& Al-Marzouk N (2001) Bone density measured by dual-energy X-ray absorptiometry in healthy Kuwaiti women. Calcif Tissue Int 68, 225-229.

58. Naeem Z (2010) Vitamin D deficiency - an ignored epidemic. Int J Health Sci (Qassim) 4, 5-6.

59. Moshfegh A, Goldman J \& Cleveland L (2005) What We Eat in America, NHANES 2001-2002: Usual Nutrient Intakes from Food Compared to Dietary Reference Intakes). Beltsville, MD: US Department of Agriculture, Agricultural Research Service; available at http://www.ars.usda.gov/ SP2UserFiles/Place/12355000/pdf/0102/usualintaketables 2001-02.pdf

60. Al Mousa Z, Prakash P, Jackson $\mathrm{R}$ et al. (2003) A comparison of selected nutrient intakes in anemic and non anemic adolescent girls in Kuwait. Nutr Res 23, 425-433.

61. Forster-Coull L, Milne RL \& Barr SI (2004) British Columbia Nutrition Survey. Report on Energy and Nutrient Intakes. Victoria, BC: Ministry of Health Services; available at http://www.health.gov.bc.ca/library/publications/year/2004/ nutrientsreport.pdf

62. Fryar CD, Wright JD, Eberhardt MS et al. (2012) Trends in Nutrient Intakes and Chronic Health Conditions Among Mexican-American Adults, a 25-year Profile: United States, 1982-2006. National Health Statistics Reports no. 50. http:// www.cdc.gov/nchs/data/nhsr/nhsr050.pdf (accessed January 2012). 